\title{
GLUING SILTING OBJECTS
}

\author{
QUNHUA LIU, JORGE VITÓRIA, AND DONG YANG
}

\begin{abstract}
Recent results by Keller and Nicolás and by Koenig and Yang have shown bijective correspondences between suitable classes of t-structures and cot-structures with certain objects of the derived category: silting objects. On the other hand, the techniques of gluing (co-)t-structures along a recollement play an important role in the understanding of derived module categories. Using the above correspondence with silting objects, we present explicit constructions of gluing of silting objects, and, furthermore, we answer the question of when the glued silting is tilting.
\end{abstract}

\section{$\S 1$. Introduction}

In a given triangulated category, the study of its torsion pairs helps to understand its structure. Two kinds of torsion pairs have been considered with particular emphasis in the literature. These are the notions of t-structure introduced by Beilinson, Bernstein, and Deligne in [5] and co-tstructure introduced independently by Bondarko in [8] and by Pauksztello in [22]. These are torsion pairs with an additional property concerning the suspension functor of the underlying triangulated category, and they give rise to additive (or even abelian in the case of t-structures) subcategories which are of interest. In this paper, we work with correspondences that classify these torsion pairs in terms of objects of the triangulated category.

Keller and Vossieck established in [17] a bijection between bounded tstructures and equivalence classes of silting objects in the bounded derived category of modules over the path algebra of a Dynkin quiver over a field. Recently, this bijection has been extended by Keller and Nicolás in [16] for the bounded derived categories of homologically smooth nonpositive differential graded algebras, and by Koenig and Yang in [18] for bounded derived categories of finite-dimensional algebras over a field. Indeed, they show that in such a category, there is a bijection between silting objects

Received July 10, 2012. Revised November 10, 2013. Accepted February 5, 2014.

First published online January 9, 2015.

2010 Mathematics Subject Classification. Primary 16E35; Secondary 18 E30.

(C) 2014 by The Editorial Board of the Nagoya Mathematical Journal 
and bounded t-structures whose hearts are length categories. It turns out that Keller and Vossieck's result is a corollary, since for algebras of finite representation type all hearts of bounded t-structures are length categories. A new correspondence between silting objects and bounded co-t-structures was proved in [21] and [16]. This bijection will be central in our approach.

Silting objects play, thus, a more general role than tilting objects. They describe all hearts which are length categories and these turn out to be precisely those which are module categories over some finite-dimensional algebra over a field. This algebra, although not in general derived equivalent to the one we started with, is obtained as the endomorphism algebra of the silting object, just like in the tilting setting. Indeed, it is easy to observe that a silting object is tilting if and only if it lies in the heart of the corresponding t-structure.

Gluing techniques with respect to a recollement, due to Beilinson, Bernstein, and Deligne, have been intensively studied in [5] for t-structures and, recently, in [8] for co-t-structures. This leads to the natural question of how to glue silting objects and which silting objects are glued from smaller ones. Indeed, recent work by the two first authors has shown that, in the piecewise hereditary case, all bounded t-structures whose heart is a length category are glued with respect to a nontrivial recollement (see [20]). In this setting it is then clear that every silting object can be decomposed by this process into as many pieces as derived simple factors of the algebra (check [3] and [4] for terminology). It turns out, however, that an answer to the problem of gluing silting objects can be given more easily when the focus is on co-t-structures rather than on t-structures. Our main result is as follows.

TheOREM A (Theorem 3.1). Let $\mathcal{R}$ be a recollement of a triangulated category $\mathcal{D}$ of the form

$$
\mathcal{Y} \underset{i^{!}}{\stackrel{i^{*}}{<}} \mathcal{i} \underset{j_{*}}{\stackrel{j^{*}}{<}} \mathcal{X} .
$$

Let $X$ and $Y$ be, respectively, silting objects of $\mathcal{X}$ and $\mathcal{Y}$, and let $\left(\mathcal{X}_{\geq 0}, \mathcal{X}_{\leq 0}\right)$ and $\left(\mathcal{Y}_{\geq 0}, \mathcal{Y}_{\leq 0}\right)$ be, respectively, the associated co-t-structures in $\mathcal{X}$ and $\mathcal{Y}$. Then the induced co-t-structure $\left(\mathcal{D}_{\geq 0}, \mathcal{D}_{\leq 0}\right)$ in $\mathcal{D}$ is associated with the silting object $Z=i_{*} Y \oplus K_{X}$ in $\mathcal{D}$, with $K_{X}$ defined by the triangle

$$
i_{*} \beta_{\geq 1} i^{!} j_{!} X \rightarrow j_{!} X \rightarrow K_{X} \rightarrow\left(i_{*} \beta_{\geq 1} i^{!} j_{!} X\right)[1],
$$

where $\beta_{\geq 1}$ is a (nonfunctorial) choice of truncation for the co-t-structure $\left(\mathcal{Y}_{\geq 0}, \mathcal{Y}_{\leq 0}\right)$ in $\mathcal{Y}$. 
Furthermore, the question of when we can glue derived equivalencesthat is, tilting objects - comes as a particular setting of the general context of gluing silting. Similar constructions of tilting objects have been discussed in [19] and [2]. In particular, we will show that the construction in [2] is a particular case of the preceding construction. The following is our main theorem concerning tilting (see Theorem 4.5).

TheOREM B (Theorem 4.5). Let $\mathcal{R}$ be a recollement of $\mathcal{D}=\mathcal{D}^{b}(R)$ by $\mathcal{X}=$ $\mathcal{D}^{b}(C)$ and $\mathcal{Y}=\mathcal{D}^{b}(B)$, where $R, C$, and $B$ are finite-dimensional algebras over a field of finite global dimension. Let $X$ and $Y$ be tilting objects of $\mathcal{X}$ and $\mathcal{Y}$, respectively. Then $Z=i_{*} Y \oplus K_{X}$ is tilting in $\mathcal{D}$ if and only if the following conditions hold:

(a) $\operatorname{Hom}_{\mathcal{Y}}\left(Y, i^{*} j_{*} X[k]\right)=0$ for all $k<-1$,

(b) $\operatorname{Hom}_{\mathcal{Y}}\left(i^{*} j_{*} X, Y[k]\right)=0$ for all $k<0$,

(c) $\operatorname{Hom}_{\mathcal{Y}}\left(i^{*} j_{*} X, i^{*} j_{*} X[k]\right)=0$ for all $k<-1$.

This paper is structured as follows. In the next section we discuss some preliminary results on recollements, t-structures, co-t-structures, and silting needed for the later sections. In Section 3 we show how to glue silting objects and we use this in Section 4 to give necessary and sufficient conditions for the glued silting to be tilting. These conditions are particularly nice in the hereditary case and they are made explicit in Section 5. Finally, in Section 6, we make a few observations on the gluing of Happel-Reiten-Smalø (HRS)tilts with a view towards a result on the compatibility of silting mutation with gluing (see Corollary 6.9).

\section{§2. Preliminaries}

Throughout, $\mathbb{K}$ denotes a fixed field, and $R, B$, and $C$ denote finitedimensional $\mathbb{K}$-algebras. The bounded derived category of finitely generated right $R$-modules and the bounded homotopy category of finitely generated projective right $R$-modules will be denoted, respectively, by $\mathcal{D}^{b}(R)$ and $\mathcal{K}^{b}(\operatorname{proj}-R)$. The symbols $\mathcal{X}, \mathcal{Y}$, and $\mathcal{D}$ denote triangulated categories.

\subsection{Recollements}

A recollement of $\mathcal{D}$ by $\mathcal{X}$ and $\mathcal{Y}$ is a diagram of six triangle functors

$$
\mathcal{Y} \underset{i^{!}}{\stackrel{i_{*}}{\leftarrow}} \mathcal{i} \underset{i^{*}}{\stackrel{j_{*}}{<}} \mathcal{亡}
$$

satisfying the following properties: 
(1) $\left(i^{*}, i_{*}\right),\left(i_{*}, i^{!}\right),\left(j_{!}, j^{*}\right),\left(j^{*}, j_{*}\right)$ are adjoint pairs,

(2) $i_{*}, j_{*}, j_{\text {! }}$ are full embeddings,

(3) $i^{!} \circ j_{*}=0$ (and thus also $j^{*} \circ i_{*}=0$ and $i^{*} \circ j_{!}=0$ ),

(4) for each $Z \in \mathcal{D}$, the units and counits of the adjunctions yield triangles

$$
\begin{aligned}
& i_{*} i^{!} Z \rightarrow Z \rightarrow j_{*} j^{*} Z \rightarrow i_{*} i^{!} Z[1], \\
& j_{!} j^{*} Z \rightarrow Z \rightarrow i_{*} i^{*} Z \rightarrow j_{!} j^{*} Z[1] .
\end{aligned}
$$

The following result allows us to change the sides of a recollement.

Theorem 2.1 ([7, Propositions 3.6-3.7], [15, Proposition 5, Theorem 7]). Let $\mathcal{R}$ be a recollement of $\mathcal{D}$ of the form (2.1). If $\mathcal{D}$ has a Serre functor $S$, then both $\mathcal{X}$ and $\mathcal{Y}$ have Serre functors (resp., $S_{\mathcal{X}}$ and $S_{\mathcal{Y}}$ ) and there are reflected recollements $\mathcal{R}_{U}$ and $\mathcal{R}_{L}$ (resp., upper and lower reflection)

$$
\mathcal{R}_{U}: \mathcal{X} \underset{j !}{\stackrel{j^{\#}}{\longleftarrow}} \mathcal{D} \underset{j^{*}}{\stackrel{i_{\#}}{\longleftarrow}} \stackrel{\stackrel{i_{*}}{\longleftarrow}}{\longleftarrow} \mathcal{Y},
$$

where $i_{\#}=S^{-1} i_{*} S_{\mathcal{Y}}$ and $j^{\#}=S_{\mathcal{X}}^{-1} j^{*} S$ are left adjoints of $i^{*}$ and $j_{!}$, respectively, and

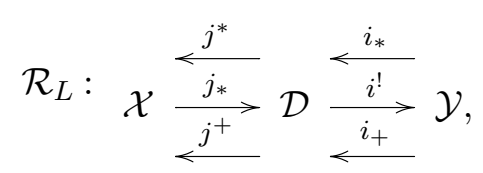

where $i_{+}=S i_{*} S_{\mathcal{Y}}^{-1}$ and $j^{+}=S_{\mathcal{X}} j^{*} S^{-1}$ are right adjoints of $i^{!}$and $j_{*}$, respectively.

\section{2. t-structures and co-t-structures}

A torsion pair in $\mathcal{D}$ is a pair of strict full subcategories $\left(\mathcal{D}^{\prime}, \mathcal{D}^{\prime \prime}\right)$, closed under taking direct summands and direct sums, such that

(1) $\operatorname{Hom}_{\mathcal{D}}(X, Y)=0$ for all $X \in \mathcal{D}^{\prime}$ and $Y \in \mathcal{D}^{\prime \prime}$,

(2) for each $Z$ in $\mathcal{D}$, there are $X \in \mathcal{D}^{\prime}, Y \in \mathcal{D}^{\prime \prime}$ and a triangle

$$
X \rightarrow Z \rightarrow Y \rightarrow X[1]
$$

By definition, it is easy to see that

$$
\begin{aligned}
\mathcal{D}^{\prime} & =\left\{X \in \mathcal{D}: \operatorname{Hom}_{\mathcal{D}}(X, Y)=0, \forall Y \in \mathcal{D}^{\prime \prime}\right\}=:{ }^{\perp} \mathcal{D}^{\prime \prime}, \\
\mathcal{D}^{\prime \prime} & =\left\{Y \in \mathcal{D}: \operatorname{Hom}_{\mathcal{D}}(X, Y)=0, \forall X \in \mathcal{D}^{\prime}\right\}=: \mathcal{D}^{\prime \perp}
\end{aligned}
$$


A $t$-structure in $\mathcal{D}$ is a pair $\left(\mathcal{D}^{\leq 0}, \mathcal{D}^{\geq 0}\right)$ such that $\left(\mathcal{D}^{\leq 0}, \mathcal{D}^{\geq 1}\right)$ is a torsion pair and $\mathcal{D}^{\leq 0} \subseteq \mathcal{D}^{\leq 1}$, where $\mathcal{D}^{\leq n}:=\mathcal{D}^{\leq 0}[-n]$ and $\mathcal{D}^{\geq n}:=\mathcal{D}^{\geq 0}[-n]$ for $n \in$ $\mathbb{Z}$. The subcategory $\mathcal{D} \leq 0$ is called the t-aisle, and $\mathcal{D}^{\geq 0}$ is called the $t$-coaisle. The heart $\mathcal{D}^{\leq 0} \cap \mathcal{D}^{\geq 0}$ is always an abelian category. We say that the heart is a length category if it has only finitely many simple objects (up to isomorphism) and each object in the heart has finite length. A t-structure is said to be bounded if it satisfies

$$
\bigcup_{n \in \mathbb{Z}} D^{\geq n}=\mathcal{D}=\bigcup_{n \in \mathbb{Z}} D^{\leq n} .
$$

For all $n \in \mathbb{Z}$, there is a right adjoint to the inclusion of the subcategory $\mathcal{D}^{\leq n}$ in $\mathcal{D}$, called the truncation at $n$ and denoted by $\tau^{\leq n}$. Similarly, there is a left adjoint to the inclusion of $\mathcal{D}^{\geq n}$, denoted by $\tau^{\geq n}$. In fact, the triangle (2.2) can be written functorially, for any $Z \in \mathcal{D}$, as

$$
\tau^{\leq 0} Z \rightarrow Z \rightarrow \tau^{\geq 1} Z \rightarrow\left(\tau^{\leq 0} Z\right)[1]
$$

Moreover, it is possible to define associated cohomological functors

$$
H^{i}: \mathcal{D} \rightarrow \mathcal{D}^{\leq 0} \cap \mathcal{D}^{\geq 0}, \quad H^{i}(X)=\left(\tau^{\leq i} \tau^{\geq i} X\right)[i], \quad \forall X \in \mathcal{D} .
$$

If a t-structure is bounded, then for any object $X \in \mathcal{D}, H^{i}(X)=0$ for all but finitely many $i \in \mathbb{Z}$. A well-known example is the standard t-structure in $\mathcal{D}^{b}(R)$. The associated cohomological functors are given by the usual cohomology of complexes in $\mathcal{D}^{b}(R)$.

Similarly, a co-t-structure in $\mathcal{D}$ is a pair $\left(\mathcal{D}_{\geq 0}, \mathcal{D}_{\leq 0}\right)$ such that $\left(\mathcal{D}_{\geq 0}, \mathcal{D}_{\leq-1}\right)$ is a torsion pair and $\mathcal{D}_{\geq 0} \subseteq \mathcal{D}_{\geq-1}$, where $\mathcal{D}_{\geq n}:=\mathcal{D}_{\geq 0}[-n]$ and $\mathcal{D}_{\leq n}:=$ $\mathcal{D}_{\leq 0}[-n]$, for all $n \in \mathbb{Z}$. The subcategory $\mathcal{D}_{\geq 0}$ is called the co-t-aisle, and $\mathcal{D}_{\leq 0}$ is called the co-t-co-aisle. The co-heart $\mathcal{D}_{\geq 0} \cap \mathcal{D}_{\leq 0}$ is not, in general, an abelian category, as in the case of t-structures, but it still has the structure of an additive category. A co-t-structure is said to be bounded if it satisfies

$$
\bigcup_{n \in \mathbb{Z}} D_{\geq n}=\mathcal{D}=\bigcup_{n \in \mathbb{Z}} D_{\leq n} .
$$

Contrarily to t-structures, the triangle obtained from the torsion pair $\left(\mathcal{D}_{\geq 0}, \mathcal{D}_{\leq-1}\right)$ is not functorial. However, a choice of $X \in \mathcal{D}_{\geq 0}$ (resp., of $Y \in \mathcal{D}_{\leq-1}$ ) as in triangle (2.2) will be denoted by $\beta_{\geq 0} Z$ (resp., by $\beta_{\leq-1} Z$ ). A well-known example (see [8], [22]) is the standard co-t-structure in $\mathcal{K}^{b}(\operatorname{proj}-R)$. Here, the nonfunctorial choices of $\beta_{\geq 0} Z$ or $\beta_{\leq 0} Z$ for some object 
$Z \in \mathcal{K}^{b}(\operatorname{proj}-R)$ are usually given by the stupid truncations, consisting in setting suitable entries of the complex $Z$ to be zero.

It is known that t-structures and co-t-structures can be glued (or induced) with respect to a recollement (see [5] for t-structures and [8] for co-tstructures). The same arguments can be used to prove the following result.

TheOREM 2.2. Let $\mathcal{R}$ be a recollement of $\mathcal{D}$ of the form (2.1). Let $\left(\mathcal{X}^{\prime}, \mathcal{X}^{\prime \prime}\right)$ and $\left(\mathcal{Y}^{\prime}, \mathcal{Y}^{\prime \prime}\right)$ be torsion pairs in $\mathcal{X}$ and $\mathcal{Y}$, respectively.

(1) There is a glued torsion pair $\left(\mathcal{D}^{\prime}, \mathcal{D}^{\prime \prime}\right)$ in $\mathcal{D}$ defined by

$\mathcal{D}^{\prime}=\left\{Z \in \mathcal{D}: j^{*} Z \in \mathcal{X}^{\prime}, i^{*} Z \in \mathcal{Y}^{\prime}\right\}, \quad \mathcal{D}^{\prime \prime}=\left\{Z \in \mathcal{D}: j^{*} Z \in \mathcal{X}^{\prime \prime}, i^{!} Z \in \mathcal{Y}^{\prime \prime}\right\}$

(2) ([5, Théorème 1.4.10]) If $\left(\mathcal{X}^{\prime}, \mathcal{X}^{\prime \prime}[1]\right)$ and $\left(\mathcal{Y}^{\prime}, \mathcal{Y}^{\prime \prime}[1]\right)$ are t-structures, then $\left(\mathcal{D}^{\prime}, \mathcal{D}^{\prime \prime}[-1]\right)$ is a t-structure in $\mathcal{D}$.

(3) $\left(\left[8\right.\right.$, Theorem 8.2.3]) If $\left(\mathcal{X}^{\prime}, \mathcal{X}^{\prime \prime}[-1]\right)$ and $\left(\mathcal{Y}^{\prime}, \mathcal{Y}^{\prime \prime}[-1]\right)$ are co-t-structures, then $\left(\mathcal{D}^{\prime}, \mathcal{D}^{\prime \prime}[1]\right)$ is a co-t-structure in $\mathcal{D}$.

(4) ([8], [20], [28]) The gluing of bounded t-structures whose heart is a length category is a bounded t-structure whose heart is a length category. Also, the gluing of bounded co-t-structures is still bounded.

If $\mathcal{D}$ admits a Serre functor $S$, if $\mathcal{R}$ is a recollement of $\mathcal{D}$ of the form (2.1), and if $\left(\mathcal{X}^{\leq 0}, \mathcal{X}^{\geq 0}\right)$ and $\left(\mathcal{Y}^{\leq 0}, \mathcal{Y}^{\geq 0}\right)$ are t-structures in $\mathcal{X}$ and $\mathcal{Y}$, respectively, then there are three naturally associated t-structures in $\mathcal{D}$, obtained by gluing these with respect to $\mathcal{R}, \mathcal{R}_{U}$, and $\mathcal{R}_{L}$-we will denote them by $\left(\mathcal{D}^{\leq 0}, \mathcal{D}^{\geq 0}\right),\left(\mathcal{D}_{\bar{U}}^{\leq 0}, \mathcal{D}_{\bar{U}}^{\geq 0}\right)$, and $\left(\mathcal{D}_{\bar{L}}^{\leq 0}, \mathcal{D}_{\bar{L}}^{\geq 0}\right)$, respectively. Similarly, given cot-structures $\left(\mathcal{X}_{\geq 0}, \mathcal{X}_{\leq 0}\right)$ and $\left(\mathcal{Y}_{\geq 0}, \mathcal{Y}_{\leq 0}\right)$ in $\mathcal{X}$ and $\mathcal{Y}$, we get three glued co-t-structures $\left(\mathcal{D}_{\geq 0}, \mathcal{D}_{\leq 0}\right),\left(\mathcal{D}_{\geq 0}^{U}, \mathcal{D}_{\leq 0}^{U}\right)$, and $\left(\mathcal{D}_{\geq 0}^{L}, \mathcal{D}_{\leq 0}^{L}\right)$.

\subsection{Correspondences with silting objects}

An object $M \in \mathcal{D}$ is silting if $\operatorname{Hom}_{\mathcal{D}}(M, M[i])=0$, for all $i>0$, and if $\mathcal{D}$ is the smallest triangulated subcategory containing $M$ which is closed under direct summands, extensions, and shifts. It is tilting if, in addition, $\operatorname{Hom}_{\mathcal{D}}(M, M[i])=0$, for all $i<0$. We say that two such objects $M$ and $N$ are equivalent if $\operatorname{add}(M)=\operatorname{add}(N)$. The following result is a consequence of [21, Corollary 5.9] (see also [16]).

THEOREM 2.3. There is a bijection between bounded co-t-structures in $\mathcal{D}$ whose co-heart is additively generated by one object and equivalence classes of silting objects of $\mathcal{D}$. 
This bijection can be described as follows.

- Given a co-t-structure $\left(\mathcal{D}_{\geq 0}, \mathcal{D}_{\leq 0}\right)$, the associated equivalence class is given by a silting object $M$ which is an additive generator of the co-heart, that is, $\mathcal{D}_{\geq 0} \cap \mathcal{D}_{\leq 0}=\operatorname{add}(M)$.

- Given a silting object $M \in \mathcal{D}$, the associated co-t-structure is defined as follows: $\mathcal{D}_{\geq 0}$ is the smallest full subcategory closed under direct summands, direct sums, and extensions containing $\{M[i]: i \leq 0\}$; similarly, $\mathcal{D}_{\leq 0}$ is the smallest full subcategory closed under direct summands, direct sums, and extensions containing $\{M[i]: i \geq 0\}$.

TheOREM 2.4 ([18, Theorem 7.1]). In $\mathcal{D}^{b}(R)$, there are bijections between

- the set of bounded t-structures in $\mathcal{D}^{b}(R)$ whose heart is a length category;

- the set of equivalence classes of silting objects in $K^{b}(\operatorname{proj}-R)$;

- the set of bounded co-t-structures in $K^{b}(\operatorname{proj}-R)$.

Recall that $R$ is of finite global dimension if and only if $\mathcal{D}^{b}(R)$ admits a Serre functor (see [7], [23]). Also, in this case, we have $K^{b}(\operatorname{proj}-R) \cong \mathcal{D}^{b}(R)$ and, thus, the correspondence between t-structures and co-t-structures occurs in the same category. Under this assumption, the bijections of the theorem can be made explicit as follows (see [18, Section 6] for details).

- Given a t-structure $\left(\mathcal{D}^{\leq 0}, \mathcal{D}^{\geq 0}\right)$ in $\mathcal{D}^{b}(R)$, we can associate a co-t-structure $\left(\mathcal{D}_{\geq 0}, \mathcal{D}_{\leq 0}\right)$ by taking $\mathcal{D}_{\leq 0}=\mathcal{D}^{\leq 0}$ and $\mathcal{D}_{\geq 0}={ }^{\perp} \mathcal{D}^{\leq-1}$; in the language of [8], this is the left adjacent co-t-structure to the t-structure $\left(\mathcal{D}^{\leq 0}, \mathcal{D}^{\geq 0}\right)$.

- Given a co-t-structure $\left(\mathcal{D}_{\geq 0}, \mathcal{D}_{\leq 0}\right)$ in $\mathcal{D}^{b}(R)$, we can associate a t-structure by taking $\mathcal{D}^{\leq 0}=\mathcal{D}_{\leq 0}$ and $\mathcal{D}^{\geq 0}=\mathcal{D}_{\leq-1}^{\perp}$; in the language of [8], this is the right adjacent $t$-structure to the co-t-structure $\left(\mathcal{D}_{\geq 0}, \mathcal{D}_{\leq 0}\right)$.

- Given a t-structure $\left(\mathcal{D}^{\leq 0}, \mathcal{D}^{\geq 0}\right)$ in $\mathcal{D}^{b}(R)$, the associated silting object is the direct sum of the indecomposable Ext-projective objects in the aisle, that is, the objects $X$ of $\mathcal{D}^{\leq 0}$ such that $\operatorname{Hom}_{\mathcal{D}^{b}(R)}(X, Y[1])=0$ for all $Y \in \mathcal{D} \leq 0$.

- Given a silting object $M$ in $\mathcal{D}^{b}(R)$, the associated t-structure is defined as follows:

$$
\begin{aligned}
& \mathcal{D}^{\leq 0}=\left\{Z \in \mathcal{D}^{b}(R): \operatorname{Hom}_{\mathcal{D}^{b}(R)}(M, Z[i])=0, \forall i>0\right\}, \\
& \mathcal{D}^{\geq 0}=\left\{Z \in \mathcal{D}^{b}(R): \operatorname{Hom}_{\mathcal{D}^{b}(R)}(M, Z[i])=0, \forall i<0\right\} .
\end{aligned}
$$

REMARK 2.5. If $R$ is of finite global dimension, as before, then the cot-co-aisle $\mathcal{D}_{\leq 0}$ associated with a silting object $M$ in $\mathcal{D}^{b}(R)$ coincides with 
the aisle $\mathcal{D}^{\leq 0}$ associated with the same silting object. In other words, the co-t-structure associated to $M$ is left adjacent to the t-structure associated to $M$.

REMARK 2.6. The structures glued from adjacent t-structures and co-tstructures are not directly related by adjacency, as observed by Bondarko [8, Remark 8.2.4.4]. Suppose, however, that $\mathcal{D}$ has a Serre functor and that $\left(\mathcal{X}_{\geq 0}, \mathcal{X}_{\leq 0}\right)$ (resp., $\left.\left(\mathcal{Y}_{\geq 0}, \mathcal{Y}_{\leq 0}\right)\right)$ is a left adjacent co-t-structure to the t-structure $\left(\overline{\mathcal{X}}^{\leq 0}, \mathcal{X}^{\geq 0}\right)$ (resp., $\left(\mathcal{Y}^{\leq 0}, \mathcal{Y}^{\geq 0}\right)$ ). Using the descriptions provided in Theorem 2.2 and the functors of Theorem 2.1 for a fixed recollement $\mathcal{R}$, we have

$$
\mathcal{D}_{\leq 0}^{U}=\mathcal{D}^{\leq 0}=\left\{Z \in \mathcal{D}: j^{*} Z \in \mathcal{X}^{\leq 0}=\mathcal{X}_{\leq 0}, i^{*} Z \in \mathcal{Y}^{\leq 0}=\mathcal{Y}_{\leq 0}\right\} .
$$

This means that the co-structure $\left(\mathcal{D}_{\geq 0}^{U}, \mathcal{D}_{\leq 0}^{U}\right)$ glued by the upper reflected recollement $\mathcal{R}_{U}$ is left adjacent to the t-structure $\left(\mathcal{D}^{\leq 0}, \mathcal{D}^{\geq 0}\right)$ glued by the recollement $\mathcal{R}$. Similarly, the t-structure $\left(\mathcal{D}_{L}^{\leq 0}, \mathcal{D}_{L}^{\geq 0}\right)$ glued by $\mathcal{R}_{L}$ is right adjacent to the co-t-structure $\left(\mathcal{D}_{\geq 0}, \mathcal{D}_{\leq 0}\right)$ glued by $\mathcal{R}$.

REMARK 2.7. A tilting object $T$ in $\mathcal{K}^{b}(\operatorname{proj}-R)$ yields equivalences between $\mathcal{D}^{b}(R)$ and $\mathcal{D}^{b}(\operatorname{End}(T))$ and between $\mathcal{K}^{b}(\operatorname{proj}-R)$ and $\mathcal{K}^{b}(\operatorname{proj}-\operatorname{End}(T))$ (see [24, Theorem 6.4]). Under these equivalences, the t-structure and the co-t-structure associated to $T$ in $\mathcal{D}^{b}(R)$ correspond to the standard ones in $\mathcal{D}^{b}(\operatorname{End}(T))$ and $K^{b}(\operatorname{proj}-\operatorname{End}(T))$, respectively.

\subsection{HRS-tilts and silting mutation}

There are mutation operations on both t-structures and silting objects. Recall that the definition of a torsion pair in an abelian category is analogous to that of a torsion pair in a triangulated category, replacing the triangle axiom by a short exact sequence.

Theorem 2.8 ([14, Proposition 2.1], [9, Proposition 2.5]). Let $\left(\mathcal{D}^{\leq 0}, \mathcal{D}^{\geq 0}\right)$ be a bounded t-structure in a triangulated category $\mathcal{D}$ with heart $\mathcal{A}$ and associated cohomology functors $H^{i}, i \in \mathbb{Z}$. Suppose that $(\mathcal{T}, \mathcal{F})$ is a torsion pair in $\mathcal{A}$. Then $\left(\mathcal{D}_{(\mathcal{T}, \mathcal{F})}^{\leq 0}, \mathcal{D}_{(\mathcal{T}, \mathcal{F})}^{\geq 0}\right)$ is a t-structure in $\mathcal{D}$, where

$$
\begin{aligned}
& \mathcal{D}_{(\mathcal{T}, \mathcal{F})}^{\leq 0}=\left\{E \in \mathcal{D}: H^{i}(E)=0, \forall i>0, H^{0}(E) \in \mathcal{T}\right\}, \\
& \mathcal{D}_{(\mathcal{T}, \mathcal{F})}^{\geq 0}=\left\{E \in \mathcal{D}: H^{i}(E)=0, \forall i<-1, H^{-1}(E) \in \mathcal{F}\right\} .
\end{aligned}
$$

The t-structure $\left(\mathcal{D}_{(\mathcal{T}, \mathcal{F})}^{\leq 0}, \mathcal{D}_{(\mathcal{T}, \mathcal{F})}^{\geq 0}\right)$ is called the HRS-tilt of $\left(\mathcal{D}^{\leq 0}, \mathcal{D}^{\geq 0}\right)$ with respect to $(\mathcal{T}, \mathcal{F})$. 
REMARK 2.9. HRS-tilts span an important class of t-structures. In fact, it is known from [6, Theorem 3.1] and [29, Proposition 2.1] that, for a bounded t-structure $\left(\mathcal{D}^{\leq 0}, \mathcal{D}^{\geq 0}\right)$, the bounded t-structures $(\mathcal{C} \leq 0, \mathcal{C} \geq 0)$ such that $\mathcal{D}^{\leq-1} \subseteq \mathcal{C} \leq 0 \subseteq \mathcal{D}^{\leq 0}$ are precisely those obtained from $\left(\mathcal{D}^{\leq 0}, \mathcal{D}^{\geq 0}\right)$ by an HRS-tilt with respect to a torsion pair.

Assume that the heart $\mathcal{A}$ of the t-structure $\left(\mathcal{D}^{\leq 0}, \mathcal{D}^{\geq 0}\right)$ is a length category, and let $S$ be a simple object of $\mathcal{A}$ without self-extensions. The left mutation of $\left(\mathcal{D}^{\leq 0}, \mathcal{D}^{\geq 0}\right)$ with respect to $S$ is defined by

$$
\mu_{S}^{-}\left(\mathcal{D}^{\leq 0}, \mathcal{D}^{\geq 0}\right)=\left(\mathcal{D}_{-, S}^{\leq 0}, \mathcal{D}_{-, S}^{\geq 0}\right):=\left(\mathcal{D}_{\left({ }^{\perp} S, \operatorname{add}(S)\right)}^{\leq 0}, \mathcal{D}_{\left({ }^{\perp} S, \operatorname{add}(S)\right)}^{\geq 0}\right),
$$

and its right mutation by

$$
\mu_{S}^{+}\left(\mathcal{D}^{\leq 0}, \mathcal{D}^{\geq 0}\right)=\left(\mathcal{D}_{+, S}^{\leq 0}, \mathcal{D}_{+, S}^{\geq 0}\right):=\left(\mathcal{D}_{\left(\operatorname{add}(S), S^{\perp}\right)}^{\leq 0}[-1], \mathcal{D}_{\left(\operatorname{add}(S), S^{\perp}\right)}^{\geq 0}[-1]\right) .
$$

The torsion pairs $\left(\operatorname{add}(S), S^{\perp}\right)$ and $\left({ }^{\perp} S, \operatorname{add}(S)\right)$ will be called mutation torsion pairs.

On the other hand, silting mutation was introduced and studied by Buan, Reiten, and Thomas in [10] and, independently, by Aihara and Iyama in [1]. We recall its definition.

Definition 2.10. Let $M=X \oplus Y$ be a silting object in $\mathcal{D}^{b}(R)$. The left mutation of $M$ at $X$, denoted by $\mu_{X}^{-}(M)$, is defined as the direct sum $\tilde{X} \oplus Y$, where $\tilde{X}$ is the cone of a left add $(Y)$-approximation of $X$ (i.e., of a morphism $\phi: X \rightarrow L$, with $L$ in $\operatorname{add}(Y)$ such that for any $Z$ in $\operatorname{add}(Y)$, $\operatorname{Hom}_{\mathcal{D}^{b}(R)}(\phi, Z)$ is surjective).

Similarly, the right mutation of $M$ at $X$, denoted by $\mu_{X}^{+}(M)$, is defined as the sum $\bar{X} \oplus Y$, where $\bar{X}$ is the cone of a right $\operatorname{add}(Y)$-approximation of $X$ (i.e., of a morphism $\psi: K \rightarrow X$, with $K$ in $\operatorname{add}(Y)$ such that for any $Z$ in $\operatorname{add}(Y), \operatorname{Hom}_{\mathcal{D}^{b}(R)}(Z, \psi)$ is surjective).

We say that a mutation is irreducible if $X$ is indecomposable.

THEOREM 2.11 ([18, Theorem 7.12]). Irreducible silting mutations are compatible with the bijections established in Theorem 2.4. More precisely, if $S_{1}, \ldots, S_{n}$ are the simple objects in the heart of a $t$-structure $\left(\mathcal{D}^{\leq 0}, \mathcal{D}^{\geq 0}\right)$ and $X_{1}, \ldots, X_{n}$ are indecomposable Ext-projective objects in $\mathcal{D} \leq 0$ such that $\operatorname{Hom}\left(X_{i}, S_{i}\right) \neq 0$, for all $1 \leq i \leq n$, then the aisles corresponding to the silting objects $\mu_{X_{i}}^{+}(M)$ and $\mu_{X_{i}}^{-}(M)$ are $\mathcal{D}_{+, S_{i}}^{\leq 0}$ and $\mathcal{D}_{-, S_{i}}^{\leq 0}$, respectively.

The question of whether these mutations are compatible with gluing is discussed in Section 6. 


\section{$\S 3 . \quad$ Gluing of silting objects}

The results in this section make explicit in a more general setting the bijection established in [1, Theorem 2.37], for Krull-Schmidt triangulated categories $\mathcal{D}$, between the equivalence classes of silting objects in $\mathcal{D}$ containing $i_{*} Y$ as a direct summand and the equivalence classes of silting objects in the triangle quotient $\mathcal{D} / \operatorname{thick}\left(i_{*} Y\right)$. Here thick $\left(i_{*} Y\right)$ is the smallest thick triangulated subcategory of $\mathcal{D}$ containing $i_{*} Y$. More precisely, the theorem below shows how to glue two silting objects of $\mathcal{X}$ and $\mathcal{Y}$ into a silting object of $\mathcal{D}$ with respect to a recollement of the form (2.1) in a way that is compatible with the bijection between silting objects and co-t-structures of Theorem 2.3.

TheOREM 3.1. Let $\mathcal{R}$ be a recollement of $\mathcal{D}$ of the form (2.1). Let $X$ and $Y$ be silting objects corresponding to co-t-structures $\left(\mathcal{X}_{\geq 0}, \mathcal{X}_{\leq 0}\right)$ and $\left(\mathcal{Y}_{\geq 0}, \mathcal{Y}_{\leq 0}\right)$ in $\mathcal{X}$ and $\mathcal{Y}$, respectively. Then the induced co-t-structure $\left(\mathcal{D}_{\geq 0}, \mathcal{D}_{\leq 0}\right)$ in $\mathcal{D}$ is associated with the silting object $Z=i_{*} Y \oplus K_{X}$, with $K_{X}$ defined by the following triangle:

$$
i_{*} \beta_{\geq 1} i^{!} j_{!} X \rightarrow j_{!} X \rightarrow K_{X} \rightarrow\left(i_{*} \beta_{\geq 1} i^{!} j_{!} X\right)[1]
$$

where $\beta_{\geq 1}$ is a (nonfunctorial) choice of truncation for the co-t-structure $\left(\mathcal{Y}_{\geq 0}, \mathcal{Y}_{\leq 0}\right)$ in $\mathcal{Y}$.

Proof. First observe that, since $i_{*}$ is a fully faithful functor, $i_{*} Y$ is partial silting and, moreover, it is easily checked to lie in the co-heart of the glued co-t-structure $\left(\mathcal{D}_{\geq 0}, \mathcal{D}_{\leq 0}\right)$ in $\mathcal{D}$.

Let us consider the following (nonfunctorial) triangle associated with the co-t-structure in $\mathcal{Y}$

$$
i_{*} \beta_{\geq 1} i^{!} j_{!} X \rightarrow i_{*} i^{!} j_{!} X \rightarrow i_{*} \beta_{\leq 0} i^{!} j_{!} X \rightarrow\left(i_{*} \beta_{\geq 1} i^{!} j_{!} X\right)[1]
$$

and the following universal triangle of the recollement (applied to $j_{!} X$ )

$$
i_{*} i^{!} j_{!} X \rightarrow j_{!} X \rightarrow j_{*} X \rightarrow\left(i_{*} i^{!} j_{!} X\right)[1]
$$


Hence, we get the following commutative diagram where the rows are triangles

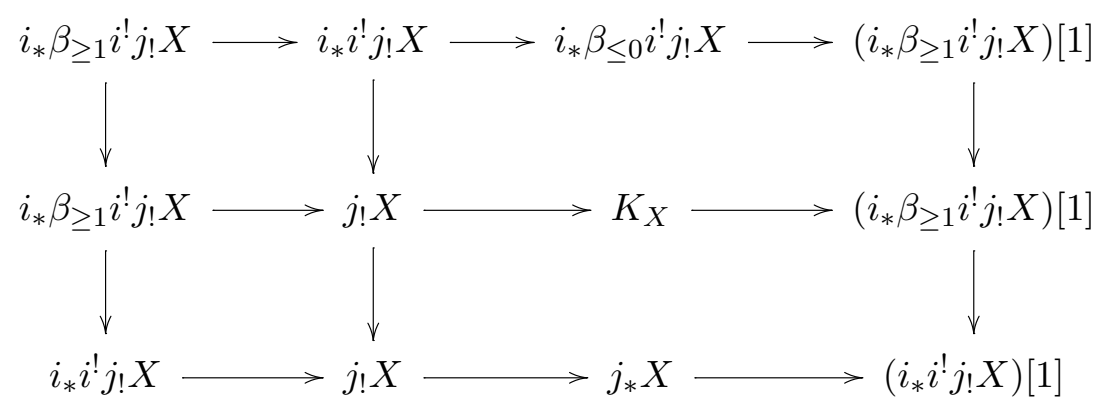

By the octahedral axiom, this can be completed to a commutative diagram

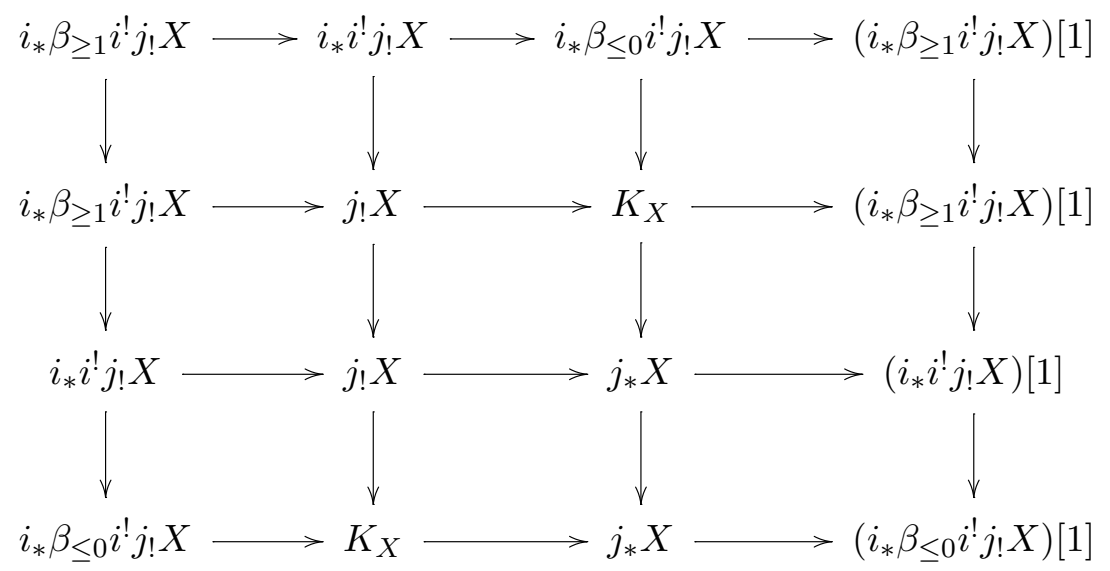

the rows of which are again triangles. We will now show that $K_{X}$ lies in the co-heart of $\left(\mathcal{D}_{\geq 0}, \mathcal{D}_{\leq 0}\right)$. Indeed, it is clear that $i_{*} \beta_{\geq 1} i^{!} j_{!} X \in \mathcal{D}_{\geq 1}$ and, therefore, that $\left(i_{*} \beta_{\geq 1} i^{!} j_{!} X\right)[1]$ lies in $\mathcal{D}_{\geq 0}$. Clearly we also have that $j_{!} X$ lies in $\mathcal{D}_{\geq 0}$ and, thus, the second row of the diagram shows that $K_{X}$ lies in $\mathcal{D}_{\geq 0}$. Similarly, since $i_{*} \beta_{\leq 0} i^{!} j_{!} X$ lies in $\mathcal{D}_{\leq 0}$ and $j_{*} X$ lies in $\mathcal{D}_{\leq 0}$, it follows that $K_{X}$ lies in $\mathcal{D}_{\leq 0}$, proving that it lies in the co-heart.

Finally, it is enough to observe that $Z$ generates $\mathcal{D}$. It is clear that $i_{*} Y$ and $j_{!} X$ generate $\mathcal{D}$ since $X$ and $Y$ generate $\mathcal{X}$ and $\mathcal{Y}$, respectively, and $i_{*}$ and $j$ ! are fully faithful functors. But, by the second triangle of the diagram, $j_{!} X$ can be generated by $K_{X}$ and an object in the image of $i_{*}$, which is generated by $i_{*} Y$. Therefore $Z$ generates $\mathcal{D}$ and thus it is silting. 
REMARK 3.2. Note that the leftmost morphism $i_{*} \beta_{\geq 1} i^{!} j_{!} X \rightarrow j_{!} X$ of a triangle in Theorem 3.1 corresponds to the chosen morphism $\beta_{\geq 1} i^{!} j ! X \rightarrow$ $i^{!} j ! X$ via the adjunction morphism

$$
\operatorname{Hom}\left(i_{*} \beta_{\geq 1} i^{!} j_{!} X, j_{!} X\right) \stackrel{\simeq}{\longrightarrow} \operatorname{Hom}\left(\beta_{\geq 1} i^{!} j_{!} X, i^{!} j_{!} X\right) .
$$

REMARK 3.3. In Krull-Schmidt categories (such as $\mathcal{D}^{b}(R)$ for an algebra, finite-dimensional over a field), the object $Z$ constructed in the proof of the theorem is not necessarily a basic object (i.e., the indecomposable summands of $Z$ can appear with multiplicities) even if both $X$ and $Y$ are basic. We can, however, obtain a basic silting object in the corresponding co-heart by ignoring the multiplicities of the direct summands of $Z$.

Similarly, we can describe a gluing of silting objects that is compatible with the bijection between silting objects and t-structures for $\mathcal{D}=\mathcal{D}^{b}(R)$, where $R$ has finite global dimension.

Corollary 3.4. Let $\mathcal{R}$ be a recollement of $\mathcal{D}=\mathcal{D}^{b}(R)$ of the form (2.1), with $\mathcal{X}=\mathcal{D}^{b}(C)$ and $\mathcal{Y}=\mathcal{D}^{b}(B)$. Let $X$ and $Y$ be silting objects corresponding to t-structures $(\mathcal{X} \leq 0, \mathcal{X} \geq 0)$ and $(\mathcal{Y} \leq 0, \mathcal{Y} \geq 0)$ in $\mathcal{X}$ and $\mathcal{Y}$, respectively. Suppose that $R$ has finite global dimension. Then the glued $t$-structure $\left(\mathcal{D}^{\leq 0}, \mathcal{D}^{\geq 0}\right)$ in $\mathcal{D}$ is associated with the silting object $Z=j_{!} X \oplus K_{Y}$, with $K_{Y}$ defined by the triangle

$$
j_{!} \alpha_{\geq 1} j^{*} i_{\#} Y \rightarrow i_{\#} Y \rightarrow K_{Y} \rightarrow\left(j_{!} \alpha_{\geq 1} j^{*} i_{\#} Y\right)[1]
$$

where $\alpha_{\geq 1}$ is a (nonfunctorial) choice of truncation for the left adjacent co-t-structure of the t-structure $(\mathcal{X} \leq 0, \mathcal{X} \geq 0)$ in $\mathcal{X}$.

Proof. This follows from Theorem 3.1 by observing, as in Remark 2.6 that $\mathcal{D}_{\leq 0}^{U}=\mathcal{D}^{\leq 0}$ and that the left adjacent co-t-structure of a t-structure corresponds to the same silting object.

The following definition settles what we will mean by gluing silting objects. As shown by Theorem 3.1, it is more natural, in this setting, to consider co-t-structures rather than t-structures.

Definition 3.5. Let $\mathcal{D}, \mathcal{X}, \mathcal{Y}$ be triangulated categories, and let $\mathcal{R}$ be a recollement of the form (2.1). We say that a silting object $Z \in \mathcal{D}$ is glued from $X \in \mathcal{X}$ and $Y \in \mathcal{Y}$ with respect to $\mathcal{R}$ if $Z$ is obtained by the construction of Theorem 3.1, that is, $Z$ corresponds to the co-t-structure glued from the co-t-structures associated to $X$ and $Y$ with respect to $\mathcal{R}$. 
The object $K_{X}$ of Theorem 3.1 can be described in a nonconstructive way as follows.

Proposition 3.6. Let $\mathcal{R}$ be a recollement of a triangulated category $\mathcal{D}$ of the form (2.1). Let $X$ and $Y$ be silting objects corresponding to co-tstructures $\left(\mathcal{X}_{\geq 0}, \mathcal{X}_{\leq 0}\right)$ and $\left(\mathcal{Y}_{\geq 0}, \mathcal{Y}_{\leq 0}\right)$ in $\mathcal{X}$ and $\mathcal{Y}$, respectively. Let $Z=$ $i_{*} Y \oplus K_{X}$ be the silting object in $\mathcal{D}$ glued from $X$ and $Y$ with respect to $\mathcal{R}$, compatible with the glued co-t-structure $\left(\mathcal{D}_{\geq 0}, \mathcal{D}_{\leq 0}\right)$. Then the following hold.

(1) Object $K_{X}$ is a right $\left(\mathcal{D}_{\geq 0} \cap \mathcal{D}_{\leq 0}\right)$-approximation of $j_{*} X$.

(2) Object $K_{X}$ is a left $\left(\mathcal{D}_{\geq 0} \cap \mathcal{D}_{\leq 0}\right)$-approximation of $j_{!} X$.

(3) Up to summands in $\mathcal{D}_{\geq 0} \cap \mathcal{D}_{\leq 0}, K_{X}$ is uniquely determined by the following conditions:

(i) $j^{*} K_{X}=X$,

(ii) $i^{*} K_{X} \in \mathcal{Y}_{\geq 0}$,

(iii) $i^{!} K_{X} \in \mathcal{Y}_{\leq 0}$.

Proof. To prove (1), let $C$ be an object of $\mathcal{D}_{>0} \cap \mathcal{D}_{\leq 0}$, and let $f$ be a morphism in $\operatorname{Hom}_{\mathcal{D}}\left(C, j_{*} X\right)$. Since $\left(i_{*} \beta_{\leq 0} i^{!} j_{!} X\right)[1]$ lies in $\mathcal{D}_{\leq-1}$, it is clear that $\operatorname{Hom}_{\mathcal{D}}\left(C,\left(i_{*} \beta_{\leq 0} i^{!} j_{!} X\right)[1]\right)=0$ and thus, by using the defining triangle

$$
i_{*} \beta_{\leq 0} i^{!} j_{!} X \rightarrow K_{X} \rightarrow j_{*} X \rightarrow\left(i_{*} \beta_{\leq 0} i^{!} j_{!} X\right)[1]
$$

$f$ factors through $K_{X}$, proving (1). Analogously, (2) can be shown using the defining triangle

$$
i_{*} \beta_{\geq 1} i^{!} j_{!} X \rightarrow j_{!} X \rightarrow K_{X} \rightarrow\left(i_{*} \beta_{\geq 1} i^{!} j_{!} X\right)[1]
$$

In order to prove (3) observe that, for any object $L$ satisfying properties (i), (ii), and (iii), we have a canonical triangle coming from the recollement $\mathcal{R}$

$$
i_{*} i^{!} L \rightarrow L \rightarrow j_{*} X \rightarrow i_{*} i^{!} L[1]
$$

and, since $i_{*}\left(\mathcal{Y}_{\leq 0}\right) \subseteq \mathcal{D}_{\leq 0}$, we have that $i_{*} i^{!} L[1]$ lies in $\mathcal{D}_{\leq-1}$. Therefore, for any object $C$ in $\mathcal{D}_{\geq 0} \cap \mathcal{D}_{\leq 0}$, any map from $C$ to $j_{*} X$ factors through $L$, proving that $L$ is a right $\left(\mathcal{D}_{\geq 0} \cap \mathcal{D}_{\leq 0}\right)$-approximation of $j_{*} X$. Hence, by (1), $L$ differs from $K_{X}$ by a summand in $\mathcal{D}_{\geq 0} \cap \mathcal{D}_{\leq 0}$. 


\section{$\S 4$. Gluing of tilting objects}

In this section we investigate necessary and sufficient conditions for the glued silting to be tilting. These conditions will be expressed exclusively in terms of the functors of the recollement and of vanishing conditions on $\mathcal{X}$ and $\mathcal{Y}$ rather than in $\mathcal{D}$. In this section, $R$ has finite global dimension and, thus, $\mathcal{D}^{b}(R)$ has a Serre functor. Under this assumption we can use Theorem 2.1 and the functors therein.

Proposition 4.1. Let $\mathcal{R}$ be a recollement of $\mathcal{D}=\mathcal{D}^{b}(R)$ of the form (2.1), with $\mathcal{X}=\mathcal{D}^{b}(C)$ and $\mathcal{Y}=\mathcal{D}^{b}(B)$. Let $X$ and $Y$ be silting objects of $\mathcal{X}$ and $\mathcal{Y}$, respectively. Then $Z=i_{*} Y \oplus K_{X}$ is tilting if and only if the following conditions are satisfied:

(1) $Y$ is tilting,

(2) $\operatorname{Hom}_{\mathcal{Y}}\left(Y, i^{!} j_{!} X[k]\right)=0$ for all $k<0$,

(3) $\operatorname{Hom}_{\mathcal{Y}}\left(i^{*} j_{*} X, Y[k]\right)=0$ for all $k<0$,

(4) $\operatorname{Hom}_{\mathcal{X}}\left(X, j^{+} K_{X}[k]\right)=0$ for all $k<0$.

Proof. The induced silting object $Z$ corresponds to the co-t-structure glued along $\mathcal{R}$ and to the t-structure glued along $\mathcal{R}_{L}$ from the respective structures on $\mathcal{X}$ and $\mathcal{Y}$ associated with $X$ and $Y$, respectively. The statement that $Z$ is tilting is equivalent to the statement that $Z$ lies in the heart of $\left(\mathcal{D}_{L}^{\leq 0}, \mathcal{D}_{\bar{L}}^{\geq 0}\right)$ which, by the description of the glued structures, translates into the conditions

(i) $i^{!} Z \in \mathcal{Y} \leq 0 \cap \mathcal{Y} \geq 0$ or, equivalently, $Y, i^{!} K_{X} \in \mathcal{Y} \leq 0 \cap \mathcal{Y} \geq 0$,

(ii) $j^{*} Z \in \mathcal{X} \leq 0$,

(iii) $j^{+} Z \in \mathcal{X} \geq 0$.

To examine these conditions we will use the following triangle defining $K_{X}$

$$
i_{*} \beta_{\leq 0} i^{!} j_{!} X \rightarrow K_{X} \rightarrow j_{*} X \rightarrow\left(i_{*} \beta_{\leq 0} i^{!} j_{!} X\right)[1] .
$$

Condition (i) is equivalent to $Y$ being tilting (corresponding to condition (1) of the proposition) and $i^{!} K_{X}$ lying in $\mathcal{Y}^{\leq 0} \cap \mathcal{Y} \geq 0$. By applying $i^{!}$to the triangle (4.1), we get that $i^{!} K_{X} \cong \beta_{\leq 0} i^{!} j_{!} X$ lies in $\mathcal{Y}_{\leq 0}=\mathcal{Y} \leq 0$. By Remark 2.7, $\beta_{\leq 0}$ corresponds to the stupid truncation in $\mathcal{D}^{b}(\operatorname{End}(Y))$ and, therefore, the fact that $i^{!} K_{X}$ lies in $\mathcal{Y}^{\leq 0} \cap \mathcal{Y}^{\geq 0}$ is equivalent to the condition that $i^{!} j_{!} X$ lies in $\mathcal{Y} \geq 0$. This can then be translated to condition (2) of the proposition, by definition of the t-structure associated with a silting object.

Clearly, since $j^{*}$ maps the co-heart in $\mathcal{D}$ to the co-heart in $\mathcal{X}$, (ii) is automatically satisfied for any silting $Z$ and, thus, this condition is irrelevant. 
Condition (iii) is equivalent to $\operatorname{Hom}_{\mathcal{X}}\left(X, j^{+} Z[k]\right)=\operatorname{Hom}_{\mathcal{D}}\left(j_{*} X, Z[k]\right)=0$ for all $k \leq 0$. By splitting $Z$ into its summands $i_{*} Y$ and $K_{X}$ and applying adjunction, we get precisely conditions (3) and (4) of the proposition.

REMARK 4.2. To simplify our notation, we will use the fact (see [5, Section 1.4]) that $i^{*} j_{*}=i^{!} j_{!}[1]$.

In many important cases, the left side of the recollement is just given by $\mathcal{D}^{b}(\mathbb{K})$.

Corollary 4.3. Let $\mathcal{R}$ be a recollement of $\mathcal{D}=\mathcal{D}^{b}(R)$ of the form (2.1), with $\mathcal{X}=\mathcal{D}^{b}(C)$ and $\mathcal{Y}=\mathcal{D}^{b}(\mathbb{K})$. Let $X$ be a silting object in $\mathcal{X}$, and let $Y=\mathbb{K}$. If $Z=i_{*} Y \oplus K_{X}$ is tilting, then there are finite-dimensional $\mathbb{K}$-vector spaces $X_{-1}^{\prime}, X_{0}^{\prime}$ such that $i^{*} j_{*} X \cong X_{-1}^{\prime}[1] \oplus X_{0}^{\prime}$.

Proof. In view of Remark 4.2, condition (2) of Proposition 4.1 is equivalent to $H^{k}\left(i^{*} j_{*} X\right)=0$ for all $k<-1$, since $Y=B=\mathbb{K}$. Since the Serre functor of $\mathcal{D}^{b}(\mathbb{K})$ is isomorphic to the identity functor, condition (3) of Proposition 4.1 is equivalent to $\operatorname{Hom}_{\mathcal{Y}}\left(Y[k], i^{*} j_{*} X\right)=0$ for all $k<0$, which is equivalent to $H^{k}\left(i^{*} j_{*} X\right)=0$ for all $k>0$. To summarize, if $Z$ is a tilting object, then it follows from Proposition 4.1 that $H^{k}\left(i^{*} j_{*} X\right)=0$ for $k \neq-1,0$, thus proving the corollary.

EXAMPLE 4.4. Let $R$ be the $\mathbb{K}$-algebra given by the quiver with relations

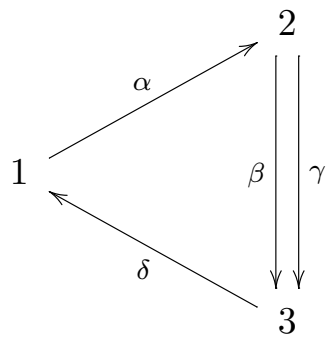

$\beta \alpha, \quad \alpha \delta, \quad \delta \gamma$

The simple module $S_{1}$ supported at 1 is a partial tilting module of projective dimension 2. It has a minimal projective resolution over $R$ given by the exact sequence

$$
0 \rightarrow P_{2} \stackrel{\beta}{\longrightarrow} P_{3} \stackrel{\delta}{\longrightarrow} P_{1} \rightarrow S_{1} \rightarrow 0 .
$$

Rickard and Schofield showed in [25] that $S_{1}$ cannot be completed to a tilting module over $R$. We will strengthen this result by showing that $S_{1}$ cannot be completed to a tilting object in $\mathcal{D}^{b}(R)$. 
If $e$ is the idempotent $e_{2}+e_{3}$ then, as a right $A$-module, $R / R e R$ is isomorphic to $S_{1}$, which does not have self-extensions. It therefore follows from [11, Theorem 3.1] and [12, Section 2] that there is a recollement

$$
\mathcal{D}^{b}(R / R e R) \underset{i^{!}}{\stackrel{i_{*}^{*}}{<}} \mathcal{D}^{b}(R) \underset{j_{*} \underset{j_{*}}{\longleftarrow}}{<} \mathcal{D}^{b}(e R e),
$$

where

$$
\begin{aligned}
& i^{*}=-\bigotimes_{R}^{\mathbb{L}} R / R e R, \quad j_{!}=-\bigotimes_{e R e}^{\mathbb{L}} e R, \\
& i_{*}=-\bigotimes_{R / R e R}^{\mathbb{L}} R / R e R, \quad j^{*}=-\bigotimes_{R}^{\mathbb{L}} R e, \\
& i^{!}=\mathbb{R H o m}_{R}(R / R e R,-), \quad j_{*}=\mathbb{R H o m}_{e R e}(R e,-) .
\end{aligned}
$$

Fix $Y=R / R e R$, and let $X \in \mathcal{D}^{b}(e R e)$ be a silting object (e.g., $X=e R e$ ). Then Theorem 3.1 yields a completion $Z=i_{*} Y \oplus K_{X}$ of the partial silting object $i_{*} Y=S_{1}$ into a silting object. However, as we will show, $Z$ is never tilting and, thus, $S_{1}$ cannot be completed to a tilting object.

Let $T$ be a basic tilting object of $\mathcal{D}^{b}(R)$ which contains $S_{1}$ as a direct summand. The functor $j^{*}$ factors through the canonical projection functor $\pi: \mathcal{D}^{b}(R) \rightarrow \mathcal{D}^{b}(R) / \operatorname{thick}\left(S_{1}\right)$, as follows:

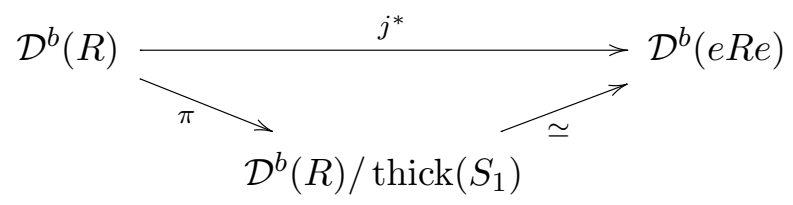

As thick $\left(S_{1}\right)$ is a silting subcategory of $\mathcal{D}^{b}(R)$, by [1, Theorem 2.37], the object $X=j^{*}(T)$ is silting in $\mathcal{D}^{b}(e R e)$ and $T$ is equivalent to $S_{1} \oplus K_{X}$. Observe that $e R e$ is the path algebra of the Kronecker quiver

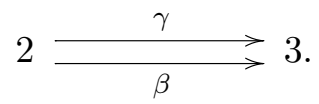


We draw the component of the AR quiver of $\mathcal{D}^{b}(e R e)$ containing the preprojective modules

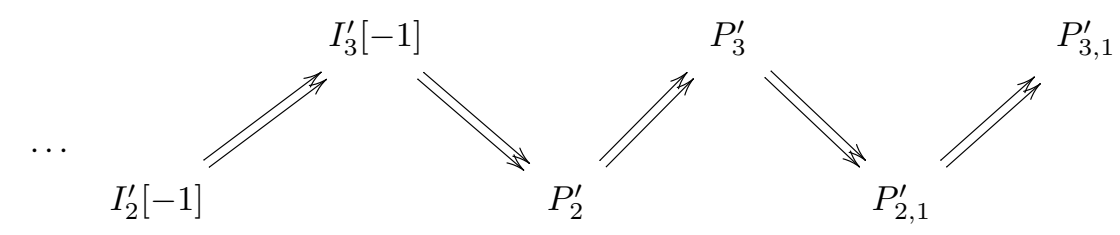

where $P_{2}^{\prime}$ and $P_{3}^{\prime}$ are, respectively, the indecomposable projective $e R e-$ module at the vertex 2 and 3. Let $\tau=\tau_{e R e}$ denote the Auslander-Reiten translation of $\mathcal{D}^{b}(e R e)$. Then according to [26, Lemma 3.1], there exist $p$ and $q$ such that $\tau^{p} P_{2}^{\prime}[q]$ is a direct summand of $K_{X}$. We claim that

$$
H^{n}\left(i^{*} j_{*}\left(\tau^{p} P_{2}^{\prime}[q]\right)\right)= \begin{cases}\mathbb{K} & \text { if } n=-q-1,-q+1 \\ 0 & \text { otherwise }\end{cases}
$$

As a consequence of Corollary 4.3, $S_{1} \oplus K_{X}$ cannot be a tilting object, which is a contradiction.

The claim follows from a direct computation, which we show only for $P_{2,1}^{\prime}=\tau^{-1} P_{2}^{\prime}$ (it is analogous for others). For $n \in \mathbb{Z}$, we have

$$
\begin{aligned}
H^{n}\left(i^{*} j_{*}\left(P_{2,1}^{\prime}\right)\right) & =H^{n+1}\left(i^{!} j_{!}\left(P_{2,1}^{\prime}\right)\right) \\
& =H^{n+1}\left(\mathbb{R} \operatorname{Hom}_{R}\left(R / R e R, j_{!}\left(P_{2,1}^{\prime}\right)\right)\right) \\
& =H^{n+1}\left(\mathbb{R H o m}_{R}\left(S_{1}, j_{!}\left(P_{2,1}^{\prime}\right)\right)\right) \\
& =\operatorname{Hom}_{\mathcal{D}^{b}(R)}\left(S_{1}, j_{!}\left(P_{2,1}^{\prime}\right)[n+1]\right) .
\end{aligned}
$$

We take a minimal projective resolution of $P_{2,1}^{\prime}$

$$
P_{2}^{\prime} \stackrel{\left(\begin{array}{l}
\gamma \\
\beta
\end{array}\right)}{\longrightarrow} \underline{P_{3}^{\prime} \oplus P_{3}^{\prime}}
$$

where the underlined term is in degree 0 . Applying $j$ ! we obtain the following object in $\mathcal{D}^{b}(R)$ :

$$
P_{2} \stackrel{\left(\begin{array}{l}
\gamma \\
\beta
\end{array}\right)}{\longrightarrow} \underline{P_{3} \oplus P_{3}}
$$

The above differential is injective and its cokernel has the following minimal injective resolution:

$$
\underline{I_{2} \oplus I_{2} \oplus I_{1}} \rightarrow I_{3} \oplus I_{3} \oplus I_{3} \rightarrow I_{1} \rightarrow I_{2} \rightarrow I_{3}
$$


Therefore $j_{!}\left(P_{2,1}^{\prime}\right)$ is isomorphic to this complex in $\mathcal{D}^{b}(R)$ and it follows that

$$
\operatorname{Hom}_{R}\left(S_{1}, j_{!}\left(P_{2,1}^{\prime}\right)[n+1]\right)= \begin{cases}\mathbb{K} & \text { if } n=-1,1 \\ 0 & \text { otherwise }\end{cases}
$$

In using Proposition 4.1 we do not seem to be dealing with computations in $\mathcal{D}$. Still, these occur in the construction of $K_{X}$. This can be avoided by introducing the assumption that $X$ is tilting.

THEOREM 4.5. Let $\mathcal{R}$ be a recollement of $\mathcal{D}=\mathcal{D}^{b}(R)$ of the form (2.1), with $\mathcal{X}=\mathcal{D}^{b}(C)$ and $\mathcal{Y}=\mathcal{D}^{b}(B)$. Let $X$ and $Y$ be tilting objects of $\mathcal{X}$ and $\mathcal{Y}$, respectively. Then $Z=i_{*} Y \oplus K_{X}$ is tilting in $\mathcal{D}$ if and only if the following conditions are satisfied:

(a) $\operatorname{Hom}_{\mathcal{Y}}\left(Y, i^{*} j_{*} X[k]\right)=0$ for all $k<-1$,

(b) $\operatorname{Hom}_{\mathcal{Y}}\left(i^{*} j_{*} X, Y[k]\right)=0$ for all $k<0$,

(c) $\operatorname{Hom}_{\mathcal{Y}}\left(i^{*} j_{*} X, i^{*} j_{*} X[k]\right)=0$ for all $k<-1$.

Proof. Condition (a) is obtained from Proposition 4.1(2) and from Remark 4.2, while condition (b) corresponds exactly to Proposition 4.1(3). We will show that if $X$ is tilting and (a) and (b) are satisfied, then Proposition 4.1(4) is equivalent to (c). Indeed, if $X$ is tilting, $\operatorname{Hom}_{\mathcal{D}}\left(j_{*} X, j_{*} X[k]\right)=0$ for all $k \neq 0$ and thus, applying the functor $\operatorname{Hom}_{\mathcal{D}}\left(j_{*} X,{ }_{-}\right)$to the $k$ th shift of triangle (4.1), we get $\operatorname{Hom}_{\mathcal{D}}\left(j_{*} X, K_{X}[k]\right) \cong \operatorname{Hom}_{\mathcal{D}}\left(j_{*} X,\left(i_{*} \beta_{\leq 0} i^{!} j_{!} X\right)[k]\right)$ for all $k \neq 0,1$. Since $\left(j_{*}, j^{+}\right)$is an adjoint pair, this shows that Proposition 4.1(4) is equivalent to

$$
\operatorname{Hom}_{\mathcal{D}}\left(j_{*} X,\left(i_{*} \beta_{\leq 0} i^{!} j_{!} X\right)[k]\right)=\operatorname{Hom}_{\mathcal{D}}\left(i^{*} j_{*} X,\left(\beta_{\leq 0} i^{!} j_{!} X\right)[k]\right)=0, \quad \forall k<0 .
$$

Applying the functor $\operatorname{Hom}_{\mathcal{Y}}\left(i^{*} j_{*} X,_{-}\right)$to the triangle

$$
\left(\beta_{\geq 1} i^{!} j_{!} X\right)[k] \rightarrow i^{!} j_{!} X[k] \rightarrow\left(\beta_{\leq 0} i^{!} j_{!} X\right)[k] \rightarrow\left(\beta_{\geq 1} i^{!} j_{!} X\right)[k+1]
$$

we get that $\operatorname{Hom}_{\mathcal{Y}}\left(i^{*} j_{*} X, \beta_{\leq 0} i^{!} j_{!} X[k]\right) \cong \operatorname{Hom}_{\mathcal{Y}}\left(i^{*} j_{*} X, i^{!} j_{!} X[k]\right)$, for all $k<0$. Indeed, this follows from (b) after recalling that $\beta_{\geq 1} i^{!} j_{!} X$ lies in $\mathcal{Y}_{\geq 1}$, which by construction (see Section 2.3 ) is suitably generated by $(Y[n])_{n<0}$, showing that $\operatorname{Hom}_{\mathcal{Y}}\left(i^{*} j_{*} X,\left(\beta_{\geq 1} i^{!} j_{!} X\right)[k+1]\right)=0$ for all $k<0$. Using again Remark 4.2, we obtain condition (c), thus finishing the proof.

Before showing applications of this theorem, we discuss the behavior of the Serre functor with respect to a t-structure corresponding to a tilting object. Recall from [5, Proposition 3.1.10] that for any bounded tstructure $\left(\mathcal{D}^{\leq 0}, \mathcal{D}^{\geq 0}\right)$ in $\mathcal{D}^{b}(R)$ with heart $\mathcal{A}$, there is a triangle functor real : 
$\mathcal{D}^{b}(\mathcal{A}) \rightarrow \mathcal{D}^{b}(R)$, t-exact for the standard t-structure $\left(\mathcal{D}_{\mathrm{st}}^{\leq 0}, \mathcal{D}_{\mathrm{st}}^{\geq 0}\right)$ in $\mathcal{D}^{b}(\mathcal{A})$ and $\left(\mathcal{D}^{\leq 0}, \mathcal{D}^{\geq 0}\right)$ in $\mathcal{D}^{b}(R)$, that is, it is both right t-exact (real $\left(\mathcal{D}_{\text {st }}^{\leq 0}\right) \subseteq \mathcal{D}^{\leq 0}$ ) and left t-exact $\left(\operatorname{real}\left(\mathcal{D}_{\mathrm{st}}^{\geq 0}\right) \subseteq \mathcal{D}^{\geq 0}\right)$.

Lemma 4.6. Let $\left(\mathcal{D}^{\leq 0}, \mathcal{D}^{\geq 0}\right)$ be a bounded $t$-structure in $\mathcal{D}^{b}(R)$ with associated basic silting object $T$. Let $S_{R}$ be the Serre functor of $\mathcal{D}^{b}(R)$. Then the following are equivalent:

(1) $T$ is tilting,

(2) $S_{R}$ is right t-exact,

(3) $S_{R}(T)$ lies in the heart.

Proof. (1) $\Rightarrow(2)$ : Suppose that $T$ is tilting. Let $\Gamma=\operatorname{End}_{\mathcal{D}^{b}(R)}(T)$, and let $\left(\mathcal{D}_{\mathrm{st}}^{\leq 0}, \mathcal{D}_{\mathrm{st}}^{\geq 0}\right)$ be the standard t-structure in $\mathcal{D}^{b}(\Gamma)$. Then, by $[27$, Theorem 6.6], the realization functor is a t-exact equivalence. Therefore, real o $S_{\Gamma}=S_{R}$ oreal (since Serre functors are unique) and $\operatorname{real}\left(\mathcal{D}_{\mathrm{st}}^{\leq 0}\right)=\mathcal{D}^{\leq 0}$. Note also that $S_{\Gamma}$ preserves the standard aisle in $\mathcal{D}^{b}(\Gamma)$ since it can be realized as a derived tensor product with a bimodule. This is enough to observe that $S_{R}$ is right t-exact, since

$$
S_{R}\left(\mathcal{D}^{\leq 0}\right)=S_{R}\left(\operatorname{real}\left(\mathcal{D}_{\mathrm{st}}^{\leq 0}\right)\right)=\operatorname{real}\left(S_{\Gamma}\left(\mathcal{D}_{\mathrm{st}}^{\leq 0}\right)\right) \subseteq \operatorname{real}\left(\mathcal{D}_{\mathrm{st}}^{\leq 0}\right)=\mathcal{D}^{\leq 0} .
$$

$(2) \Rightarrow(3)$ : For any $K \in \mathcal{D}^{\leq-1}, \operatorname{Hom}_{\mathcal{D}^{b}(R)}\left(K, S_{R} T\right)=\operatorname{Hom}_{\mathcal{D}^{b}(R)}(T, K)=0$, by definition of the t-structure associated to $T$ (see Section 2.3). This shows that $S_{R} T \in \mathcal{D}^{\geq 0}$. If $S_{R}$ is right t-exact, then $S_{R}(T) \in \mathcal{D}^{\leq 0}$ and, thus, $S_{R}(T)$ lies in the heart.

$(3) \Rightarrow(1)$ : Suppose that $S_{R}(T)$ lies in the heart. By the definition of the t-structure associated to $T$, it is clear that $\operatorname{Hom}(T, T[k])=\operatorname{Hom}(T$, $\left.S_{R}(T)[-k]\right)=0$ for all $k \neq 0$. Therefore $T$ is tilting.

Proposition 4.7. Let $\mathcal{R}$ be a recollement of $\mathcal{D}=\mathcal{D}^{b}(R)$ of the form (2.1), with $\mathcal{X}=\mathcal{D}^{b}(C)$ and $\mathcal{Y}=\mathcal{D}^{b}(B)$. Let $X$ and $Y$ be tilting objects of $\mathcal{X}$ and $\mathcal{Y}$, respectively. If $i^{*} j_{*} X$ is an element of $\mathcal{D}^{b}(B)$ such that $\operatorname{Hom}_{\mathcal{Y}}\left(Y, i^{*} j_{*} X[k]\right)$ is zero except for two consecutive values of $k \in \mathbb{Z}$, then the family of silting objects $\left(Z_{n}=i_{*} Y \oplus K_{X[n]}\right)_{n \in \mathbb{Z}}$ contains at least one tilting object.

Proof. Let $(\mathcal{Y} \leq 0, \mathcal{Y} \geq 0)$ be the t-structure associated with $Y$. By definition of the t-structure associated to $Y, \operatorname{Hom}_{\mathcal{Y}}\left(Y, i^{*} j_{*} X[k]\right)$ is nonzero exactly for two consecutive values of $k \in \mathbb{Z}$ if and only if there is $a$ in $\mathbb{Z}$ such that $i^{*} j_{*} X \in$ $\mathcal{Y} \geq a \cap \mathcal{Y} \leq a+1$. Since $Y$ is tilting, by Lemma 4.6 we have that $S_{\mathcal{Y}} i^{*} j_{*} X$ lies in $\mathcal{Y} \leq a+1$. In Theorem 4.5, condition (a) can be reformulated as $i^{*} j_{*} X \in \mathcal{Y} \geq-1$ 
and condition (b) as $S_{\mathcal{Y}} i^{*} j_{*} X \in \mathcal{Y} \leq 0$. Note that once condition (c) is satisfied for some $X[n]$, it is satisfied for all its shifts. By Theorem $4.5, Z_{n}$ is tilting if and only if

(a) $i^{*} j_{*} X[n] \in \mathcal{Y}^{\geq-1}$, which means that $a-n \geq-1$ or, equivalently, $n \leq$ $a+1$;

(b) $S_{\mathcal{Y}} i^{*} j_{*} X[n] \in \mathcal{Y} \leq 0$, which is guaranteed if $a+1-n \leq 0$ or, equivalently, $n \geq a+1$

(c) $\operatorname{Hom}_{\mathcal{Y}}\left(i^{*} j_{*} X, i^{*} j_{*} X[k]\right)=0$ for all $k<-1$.

Therefore, take $n=a+1$, and we only need to check condition (c). This condition is, however, automatic from our assumption that $i^{*} j_{*} X$ has cohomologies with respect to $\left(\mathcal{Y}^{\leq 0}, \mathcal{Y}^{\geq 0}\right)$ concentrated in exactly two consecutive degrees, thus finishing the proof.

REMARK 4.8. In the family $\left(Z_{n}\right)_{n \in \mathbb{Z}}$ there may be more than one tilting object. Let $a$ be the maximal integer such that $i^{*} j_{*} X \in \mathcal{Y} \geq a$, and let $b$ be the minimal integer such that $S_{\mathcal{Y}} i^{*} j_{*} X \in \mathcal{Y} \leq b$. Then $Z_{n}$ is tilting if and only if $b \leq n \leq a+1$.

From the proof above, we see that, for $X$ and $Y$ tilting, whenever $i^{*} j_{*} X$ lies in $\mathcal{Y}^{\leq 0} \cap \mathcal{Y}^{\geq-1}$ (i.e., the cohomologies of $i^{*} j_{*} X$ with respect to $Y$ lie in degrees -1 and 0 ), the induced silting $Z=i_{*} Y \oplus K_{X}$ is tilting. Since $i^{*} j_{*}=$ $i^{!} j_{\text {! }}[1]$, the condition is equivalent to that $i^{!} j_{!} X \in \mathcal{Y} \leq 1 \cap \mathcal{Y} \geq 0$ which, in its turn, is equivalent to $\operatorname{Hom}_{\mathcal{Y}}\left(Y, i^{!} j_{!} X[k]\right) \cong \operatorname{Hom}_{\mathcal{D}}\left(i_{*} Y, j_{!} X[k]\right)=0$ whenever $k \neq 0,1$. Under this assumption, we can apply the construction from $[2$, Theorem 2.5] to the pair $T_{1}=j_{!} X$ and $T_{2}=i_{*} Y$. A similar construction was studied in [19] in the setting of triangular matrix rings.

We briefly recall the construction in [2]. Since $\left(j_{!}, j^{*}\right)$ is an adjoint pair in the recollement (of the form (2.1)), $\operatorname{Hom}_{\mathcal{D}}\left(j_{!} X, i_{*} Y[k]\right) \cong \operatorname{Hom}_{\mathcal{X}}(X$, $\left.j^{*} i_{*} Y[k]\right)=0$ for all $k \in \mathbb{Z}$. Let $m$ be the dimension of $\operatorname{Hom}_{\mathcal{D}}\left(i_{*} Y, j_{!} X[1]\right)$, and take a basis $\alpha_{1}, \ldots, \alpha_{m}: i_{*} Y \rightarrow j_{!} X[1]$. Consider the universal maps

$$
\begin{aligned}
& \alpha=\left(\alpha_{1}, \ldots, \alpha_{m}\right)^{\operatorname{tr}}: i_{*} Y^{\oplus m} \rightarrow j_{!} X[1], \\
& \beta=\left(\alpha_{1}, \ldots, \alpha_{m}\right): i_{*} Y \rightarrow j_{!} X[1]^{\oplus m} .
\end{aligned}
$$

The map $\alpha$ is left-universal, that is, the induced map

$$
\operatorname{Hom}\left(i_{*} Y, \alpha\right): \operatorname{Hom}_{\mathcal{D}}\left(i_{*} Y, i_{*} Y^{\oplus m}\right) \rightarrow \operatorname{Hom}_{\mathcal{D}}\left(i_{*} Y, j_{!} X[1]\right)
$$

is surjective. Similarly, the map $\beta$ is right-universal, that is, the induced map

$$
\operatorname{Hom}(\beta, j ! X[1]): \operatorname{Hom}_{\mathcal{D}}\left(j_{!} X[1]^{\oplus m}, j_{!} X[1]\right) \rightarrow \operatorname{Hom}_{\mathcal{D}}\left(i_{*} Y, j_{!} X[1]\right)
$$


is surjective. Consider the triangles determined by $\alpha$ and $\beta$ :

$$
\begin{aligned}
& T_{\alpha}: j_{!} X \rightarrow C_{1} \rightarrow i_{*} Y^{\oplus m} \stackrel{\alpha}{\rightarrow} j_{!} X[1] \\
& T_{\beta}: j_{!} X^{\oplus m} \rightarrow C_{2} \rightarrow i_{*} Y \stackrel{\beta}{\rightarrow} j_{!} X[1]^{\oplus m} .
\end{aligned}
$$

Theorem 2.5 in [2] asserts that $i_{*} Y \oplus C_{1}$ and $j_{!} X \oplus C_{2}$ are tilting objects in $\mathcal{D}$.

The next proposition proves that the tilting object $i_{*} Y \oplus C_{1}$ is precisely the silting (indeed tilting) glued from $X \in \mathcal{X}$ and $Y \in \mathcal{Y}$ with respect to the recollement $\mathcal{R}$, and $j_{!} X \oplus C_{2}$ is the silting (indeed tilting) glued from $X \in \mathcal{X}$ and $Y \in \mathcal{Y}$ with respect to the upper reflection $\mathcal{R}_{U}$. As a consequence, under the conditions of Theorem 4.5, we are not only able to glue tilting objects in the original recollement $\mathcal{R}$, but also in the upper reflection $\mathcal{R}_{U}$.

Proposition 4.9. Let $\mathcal{R}$ be a recollement of $\mathcal{D}=\mathcal{D}^{b}(R)$ of the form (2.1), with $\mathcal{X}=\mathcal{D}^{b}(C)$ and $\mathcal{Y}=\mathcal{D}^{b}(B)$. Let $X$ and $Y$ be tilting objects of $\mathcal{X}$ and $\mathcal{Y}$, respectively, such that $\operatorname{Hom} \mathcal{Y}\left(Y, i^{*} j_{*} X[k]\right)=0$ for all $k \neq 0,-1$. Then, up to multiplicity, the tilting object $i_{*} Y \oplus C_{1}$ coincides with $Z=i_{*} Y \oplus K_{X}$ of Theorem 3.1, and the tilting object $j_{!} X \oplus C_{2}$ coincides with $Z_{U}=j_{!} X \oplus K_{Y}$ of Corollary 3.4.

Proof. By hypothesis, $i^{*} j_{*} X$ lies in $\mathcal{Y} \leq 0 \cap \mathcal{Y} \geq-1$, for the t-structure $\left(\mathcal{Y}^{\leq 0}, \mathcal{Y}^{\geq 0}\right)$ associated with $Y$. We will prove that $C_{1}$ satisfies the conditions (i)-(iii) for $K_{X}$ in Proposition 3.6(3). It follows then that $i_{*} Y \oplus C_{1}$ lies in the glued co-heart of $\mathcal{D}$ and that it generates $i_{*} Y \oplus j_{!} X$, thus generating the whole of $\mathcal{D}$. Therefore, up to multiplicities, it coincides with the glued silting $Z=i_{*} Y \oplus K_{X}$.

For condition (i), we apply $j^{*}$ to the defining triangle $T_{\alpha}$ of $C_{1}$ and obtain that $j^{*} C_{1}=j^{*} j_{!} X=X$, since $j^{*} i_{*}=0$. For condition (ii), we apply $i^{*}$ to $T_{\alpha}$ and obtain that $i^{*} C_{1}=i^{*} i_{*} Y^{\oplus m}=Y^{\oplus m} \in \mathcal{Y}_{\geq 0}$, since $i^{*} j_{!}=0$. For condition (iii), we apply $i^{!}$to $T_{\alpha}$ and obtain a triangle

$$
i^{!} j_{!} X \rightarrow i^{!} C_{1} \rightarrow i^{!} i_{*} Y^{\oplus m} \stackrel{i^{!} \alpha}{\longrightarrow} i^{!} j_{!} X[1]
$$

By applying $\operatorname{Hom} y(Y,-)$ to this triangle, we obtain a long exact sequence

$$
\begin{aligned}
\cdots & \rightarrow \operatorname{Hom}_{\mathcal{Y}}\left(Y, i^{!} i_{*} Y^{\oplus m}\right) \stackrel{\left(i^{!} \alpha\right)_{*}}{\longrightarrow} \operatorname{Hom}_{\mathcal{Y}}\left(Y, i^{!} j_{!} X[1]\right) \rightarrow \operatorname{Hom}_{\mathcal{Y}}\left(Y, i^{!} C_{1}[1]\right) \\
& \rightarrow \operatorname{Hom}_{\mathcal{Y}}\left(Y, i^{!} i_{*} Y[1]^{\oplus m}\right) \rightarrow \cdots
\end{aligned}
$$


For $k>0$, we have $\operatorname{Hom}_{\mathcal{Y}}\left(Y, i^{!} i_{*} Y[k]^{\oplus m}\right) \simeq \operatorname{Hom}_{\mathcal{Y}}\left(Y, Y[k]^{\oplus m}\right)=0$ (since, by assumption, $Y$ is tilting) and for $k>1, \operatorname{Hom}_{\mathcal{Y}}\left(Y, i^{!} j_{!} X[k]\right)=0$ (by our assumption on $\left.i^{*} j_{*} X=i^{!} j_{!} X[1]\right)$. Hence, $\operatorname{Hom} \mathcal{Y}\left(Y, i^{!} C_{1}[k]\right)=0$, for all $k>1$. By adjunction, there is a commutative diagram

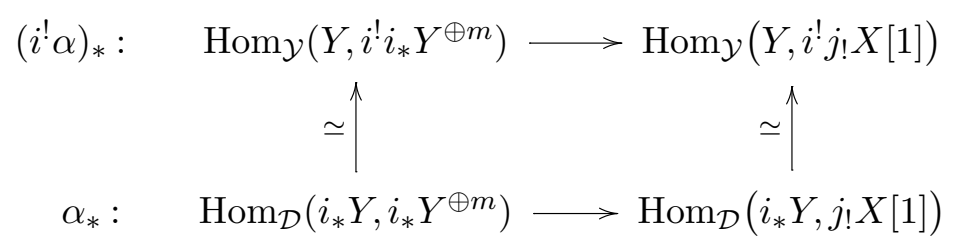

Since, by construction, $\alpha$ is left-universal, that is, $\alpha_{*}$ is surjective, it follows that $\left(i^{!} \alpha\right)_{*}$ is surjective. Hence, we get $\operatorname{Hom}_{\mathcal{Y}}\left(Y, i^{!} C_{1}[1]\right)=0$ and, thus, $i^{!} C_{1}$ lies in $\mathcal{Y}^{\leq 0}=\mathcal{Y}_{\leq 0}$. The proof for the statement about $j_{!} X \oplus C_{2}$ is analogous.

\section{$\S 5$. The hereditary case}

In this section we assume that $R$ has finite global dimension, and we apply Theorem 4.5 when $B$ is a hereditary algebra. The conditions for the glued silting to be tilting are then easier to handle.

Proposition 5.1. Let $\mathcal{R}$ be a recollement of $\mathcal{D}=\mathcal{D}^{b}(R)$ of the form (2.1), with $\mathcal{X}=\mathcal{D}^{b}(C), \mathcal{Y}=\mathcal{D}^{b}(B)$, and $B$ hereditary. Let $X$ be a tilting object in $\mathcal{X}$, and let $Y=B$. Then $Z=i_{*} Y \oplus K_{X}$ is tilting in $\mathcal{D}$ if and only if there are finitely generated $B$-modules $X_{-1}^{\prime}, X_{0}^{\prime}$, and $X_{1}^{\prime}$ such that

- $i^{*} j_{*} X$ is isomorphic to $X_{-1}^{\prime}[1] \oplus X_{0}^{\prime} \oplus X_{1}^{\prime}[-1]$,

- $X_{1}^{\prime}$ is either zero or not projective,

- $\operatorname{Hom}_{B}\left(X_{1}^{\prime}, X_{-1}^{\prime}\right)=0$.

Proof. We analyze the conditions of Theorem 4.5 when $B$ is hereditary and $Y=B$. Since $B$ is hereditary, we may assume that $i^{*} j_{*} X=$ $\bigoplus_{n \in \mathbb{Z}} X_{n}^{\prime}[-n]$, with $X_{n}^{\prime}$ a finitely generated right $B$-module for all $n$ in $\mathbb{Z}$. In this setting, $\operatorname{Hom}_{\mathcal{Y}}\left(Y, i^{*} j_{*} X[k]\right)=0$, for all $k<-1$, is equivalent to

$$
\operatorname{Hom}_{\mathcal{D}^{b}(B)}\left(B, \bigoplus_{n \in \mathbb{Z}} X_{n}^{\prime}[-n+k]\right)=0 \quad \text { for all } k<-1
$$

which happens if and only if $X_{k}^{\prime}=0$, for all $k<-1$. On the other hand, the condition

$$
\operatorname{Hom}_{\mathcal{Y}}\left(i^{*} j_{*} X, Y[k]\right)=\operatorname{Hom}_{\mathcal{D}^{b}(B)}\left(i^{*} j_{*} X, B[k]\right)=0, \quad \text { for all } k<0
$$


can be reformulated, using the Serre functor $S_{B}$ in $\mathcal{D}^{b}(B)$, by

$$
\operatorname{Hom}_{\mathcal{D}^{b}(B)}\left(B, S_{B}\left(i^{*} j_{*} X\right)[k]\right)=0, \text { for all } k>0 .
$$

It is well known that $S_{B}=[1] \circ \tau_{B}$, where $\tau_{B}$ is the Auslander-Reiten translation in $\mathcal{D}^{b}(B)$ and, thus, the statement above is equivalent to

$$
\operatorname{Hom}_{\mathcal{D}^{b}(B)}\left(B, \bigoplus_{n \in \mathbb{Z}} \tau_{B}\left(X_{n}^{\prime}\right)[-n+k+1]\right)=0, \quad \forall k>0,
$$

that is, $X_{k}^{\prime}=0$, for all $k>1$, and $X_{1}^{\prime}$ is not a projective $B$-module. So, (a) and (b) of Theorem 4.5 hold if and only if $i^{*} j_{*} X=X_{-1}^{\prime}[1] \oplus X_{0}^{\prime} \oplus X_{1}^{\prime}[-1]$, with $X_{1}^{\prime}$ not projective as a $B$-module. Assuming this, we can easily unfold the last condition of Theorem 4.5 as

$$
\operatorname{Hom}_{\mathcal{Y}}\left(i^{*} j_{*} X, i^{*} j_{*} X[k]\right)=0, \quad \forall k<-1 \quad \Leftrightarrow \quad \operatorname{Hom}_{\mathcal{D}^{b}(B)}\left(X_{1}^{\prime}, X_{-1}^{\prime}\right)=0,
$$

thus finishing the proof.

As a nice corollary, we obtain a necessary and sufficient condition for the case $\mathcal{Y}=\mathcal{D}^{b}(\mathbb{K})$.

Corollary 5.2. Let $\mathcal{R}$ be a recollement of $\mathcal{D}=\mathcal{D}^{b}(R)$ of the form (2.1), with $\mathcal{X}=\mathcal{D}^{b}(C)$ and $\mathcal{Y}=\mathcal{D}^{b}(\mathbb{K})$. Let $X$ be a tilting object in $\mathcal{X}$, and let $Y=\mathbb{K}$. Then $Z=i_{*} Y \oplus K_{X}$ is tilting if and only if there are finite-dimensional $\mathbb{K}$-vector spaces $X_{-1}^{\prime}, X_{0}^{\prime}$ such that $i^{*} j_{*} X \cong X_{-1}^{\prime}[1] \oplus X_{0}^{\prime}$.

EXAMPLE 5.3. Let $R$ be the path algebra over $\mathbb{K}$ of the quiver $1 \rightarrow 2$, of type $A_{2}$. Let $e=e_{1}$ be the trivial path at the vertex 1 . Consider the following standard recollement

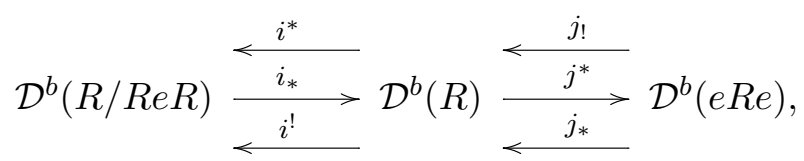

where the six functors are defined as in (4.2). Let $D$ denote the functor $\operatorname{Hom}_{\mathbb{K}}(-, \mathbb{K})$. It is easy to observe that

(1) as algebras, both $R / R e R$ and $e R e$ are isomorphic to $\mathbb{K}$;

(2) as an $R$-module, $e R$ is the simple module $S_{1}$, supported at the vertex 1 , and has an injective resolution $(D(R e) \rightarrow D(R(1-e)))$;

(3) as an $R$-module, $R / R e R$ is the simple module $S_{2}$ supported at the vertex 2 . 
Fix $Y=R / R e R$ and $X=e R e$. Then, in $\mathcal{D}^{b}(R)$, we have

$$
\begin{aligned}
i^{!} j_{!} X & =i^{!}(e R)=\mathbb{R} \operatorname{Hom}_{R}(R / R e R, e R) \\
& \cong\left(\operatorname{Hom}_{R}(R / \operatorname{Re} R, D(\operatorname{Re})) \rightarrow \operatorname{Hom}_{R}(R / \operatorname{ReR}, D(R(1-e)))\right) \\
& \cong R / \operatorname{Re} R[-1] .
\end{aligned}
$$

By Corollary 5.2, there are exactly two tilting objects in $\left\{Z_{n}=i_{*} Y \oplus K_{X[n]}\right.$ : $n \in \mathbb{Z}\}$. We construct this family using Theorem 3.1. For $n \in \mathbb{Z}$, we can choose the map $\beta_{\geq 1}\left(i^{!} j_{!} X[n]\right) \rightarrow i^{!} j_{!} X[n]$ to be

$$
\begin{cases}R / \operatorname{Re} R[n-1] \stackrel{\text { id }}{\rightarrow} R / \operatorname{Re} R[n-1] & \text { if } n \leq 0 \\ 0 \rightarrow R / \operatorname{Re} R[n-1] & \text { if } n>0 .\end{cases}
$$

By adjunction, we have a bijection

$$
\operatorname{Hom}\left(i_{*} \beta_{\geq 1}\left(i^{!} j_{!} X[n]\right), j_{!} X[n]\right) \stackrel{\simeq}{\longrightarrow} \operatorname{Hom}\left(\beta_{\geq 1}\left(i^{!} j_{!} X[n]\right), i^{!} j_{!} X[n]\right) .
$$

If $n \geq 0$, this adjunction morphism is

$$
\operatorname{Hom}_{\mathcal{D}^{b}(R)}\left(S_{2}[n-1], S_{1}[n]\right) \stackrel{\simeq}{\longrightarrow} \operatorname{Hom}_{\mathcal{D}^{b}(R)}(R / \operatorname{Re} R[n-1], R / R e R[n-1]) .
$$

Both these vector spaces are 1-dimensional over $\mathbb{K}$. Let $f$ be the preimage of $\operatorname{id}_{R / R e R[n-1]}$. Then the cone of $f[-n]$ is $(1-e) R$, the projective cover $P_{2}$ of $S_{2}$. If $n<0$, the above adjunction morphism is

$$
\operatorname{Hom}_{\mathcal{D}^{b}(R)}\left(0, S_{1}[n]\right) \stackrel{\simeq}{\longrightarrow} \operatorname{Hom}_{\mathcal{D}^{b}(R)}(0, R / R e R[n-1]) .
$$

Thus a morphism $i_{*} \beta_{\geq 1}\left(i^{!} j_{!} X[n]\right) \rightarrow j_{!} X[n]$ as in Theorem 3.1 is (see Remark 3.2)

$$
\begin{cases}S_{2}[n-1] \stackrel{f}{\rightarrow} S_{1}[n] & \text { if } n \leq 0 \\ 0 \rightarrow S_{1}[n] & \text { if } n>0\end{cases}
$$

Hence, we have

$$
K_{X[n]}=\left\{\begin{array}{ll}
P_{2}[n] & \text { if } n \leq 0, \\
S_{1}[n] & \text { if } n>0,
\end{array} \quad \text { and } \quad Z_{n}= \begin{cases}S_{2} \oplus P_{2}[n] & \text { if } n \leq 0 \\
S_{2} \oplus S_{1}[n] & \text { if } n>0\end{cases}\right.
$$

Among these silting objects, $Z_{0}=S_{2} \oplus P_{2}$ and $Z_{1}=S_{2} \oplus S_{1}[1]$ are tilting objects. 


\section{$\S 6 . \quad H R S-t i l t s$ and recollements}

In this section we show that HRS-tilts of t-structures with respect to torsion theories (see [14]) are compatible with the gluing of t-structures via recollements. The main results of this section are Theorem 6.4 and Proposition 6.5. Our notation is fixed as follows:

- $\mathcal{D}$ is a triangulated category admitting a recollement $\mathcal{R}$ of the form (2.1),

- $\left(\mathcal{Y}^{\leq 0}, \mathcal{Y}^{\geq 0}\right)$ and $(\mathcal{X} \leq 0, \mathcal{X} \geq 0)$ are bounded t-structures in $\mathcal{Y}$ and $\mathcal{X}$, respectively,

- $\left(\mathcal{D}^{\leq 0}, \mathcal{D}^{\geq 0}\right)$ is glued from $\left(\mathcal{Y}^{\leq 0}, \mathcal{Y}^{\geq 0}\right)$ and $\left(\mathcal{X}^{\leq 0}, \mathcal{X}^{\geq 0}\right)$ (see Theorem 2.2). In fact (see [5], [20]), $\left(\mathcal{D}^{\leq 0}, \mathcal{D}^{\geq 0}\right)$ is glued with respect to $\mathcal{R}$ if and only if $j_{!} j^{*}\left(\mathcal{D}^{\leq 0}\right) \subseteq \mathcal{D}^{\leq 0}$ (or, equivalently, if and only if $j_{*} j^{*}\left(\mathcal{D}^{\geq 0}\right) \subseteq \mathcal{D}^{\geq 0}$ ) in which case the restrictions to $\mathcal{Y}$ and $\mathcal{X}$ satisfy

$$
\begin{array}{ll}
\mathcal{Y} \leq 0=i^{*}\left(\mathcal{D}^{\leq 0}\right), & \mathcal{Y}^{\geq 0}=i^{!}\left(\mathcal{D}^{\geq 0}\right), \\
\mathcal{X} \leq 0=j^{*}\left(\mathcal{D}^{\leq 0}\right), & \mathcal{X} \geq 0=j^{*}\left(\mathcal{D}^{\geq 0}\right) .
\end{array}
$$

Let $\mathcal{A}_{\mathcal{D}}, \mathcal{A}_{\mathcal{Y}}$, and $\mathcal{A}_{\mathcal{X}}$ be the hearts of these t-structures. In [5, Section 1.4], it is shown that there is a recollement of abelian categories at the level of hearts

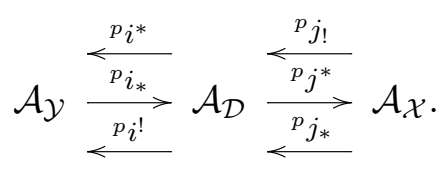

We describe these functors explicitly. Let $\epsilon_{\mathcal{D}}: \mathcal{A}_{\mathcal{D}} \hookrightarrow \mathcal{D}$ denote the full embedding (similarly $\epsilon_{\mathcal{Y}}: \mathcal{A}_{\mathcal{Y}} \hookrightarrow \mathcal{Y}$ and $\epsilon_{\mathcal{X}}: \mathcal{A}_{\mathcal{X}} \hookrightarrow \mathcal{X}$ ), and let $H_{\mathcal{D}}^{i}: \mathcal{D} \rightarrow \mathcal{A}_{\mathcal{D}}$, $i \in \mathbb{Z}$, denote the cohomological functors with respect to the fixed t-structure in $\mathcal{D}$ (similarly $H_{\mathcal{Y}}^{i}$ and $H_{\mathcal{X}}^{i}$ for the t-structures in $\mathcal{Y}$ and $\mathcal{X}$ ). Then the functors in the recollement (6.1) are given by

$$
\begin{aligned}
& p^{*}=H_{\mathcal{Y}}^{0} \circ i^{*} \circ \epsilon_{\mathcal{D}}, \quad p_{i}^{!}=H_{\mathcal{Y}}^{0} \circ i^{!} \circ \epsilon_{\mathcal{D}}, \quad p_{i_{*}}=H_{\mathcal{D}}^{0} \circ i_{*} \circ \epsilon \mathcal{Y}, \\
& { }^{p} j_{!}=H_{\mathcal{D}}^{0} \circ j_{!} \circ \epsilon_{\mathcal{X}}, \quad{ }^{p} j_{*}=H_{\mathcal{D}}^{0} \circ j_{*} \circ \epsilon_{\mathcal{X}}, \quad{ }^{p} j^{*}=H_{\mathcal{X}}^{0} \circ j^{*} \circ \epsilon_{\mathcal{D}} .
\end{aligned}
$$

See [13] for more on recollements of abelian categories.

REMARK 6.1. Since for our fixed t-structures, $i_{*}$ and $j^{*}$ are t-exact (see, e.g., [20] for details), we have that ${ }^{p} i_{*}=i_{*} \circ \epsilon \mathcal{Y}$ and ${ }^{p} j^{*}=j^{*} \circ \epsilon_{\mathcal{D}}$. As a consequence, these two functors are exact. 
Moreover, for any object $A$ in $\mathcal{A}_{\mathcal{D}}$ there are exact sequences

$$
\begin{aligned}
& 0 \rightarrow{ }^{p} i_{*} H_{\mathcal{Y}}^{-1 p} i^{*} A \rightarrow{ }^{p} j_{!}{ }^{p} j^{*} A \rightarrow A \rightarrow{ }^{p} i_{*}{ }^{p} i^{*} A \rightarrow 0, \\
& 0 \rightarrow{ }^{p} i_{*}{ }^{p} i^{!} A \rightarrow A \rightarrow{ }^{p} j_{*}{ }^{p} j^{*} A \rightarrow{ }^{p} i_{*} H_{\mathcal{Y}}^{1} i^{p} A \rightarrow 0 .
\end{aligned}
$$

Torsion pairs in $\mathcal{A}_{\mathcal{D}}$ satisfying certain conditions can be restricted to torsion pairs in $\mathcal{A}_{\mathcal{Y}}$ and $\mathcal{A}_{\mathcal{X}}$.

Lemma 6.2. Let $(\mathcal{T}, \mathcal{F})$ be a torsion pair in $\mathcal{A}_{\mathcal{D}}$. Then

(1) $\left({ }^{p} i^{*}(\mathcal{T}),{ }^{p} i^{!}(\mathcal{F})\right)$ is a torsion pair in $\mathcal{A} \mathcal{Y}$;

(2) the following are equivalent:

(i) $\left({ }^{p} j^{*}(\mathcal{T}),{ }^{p} j^{*}(\mathcal{F})\right)$ is a torsion pair in $\mathcal{A}_{\mathcal{X}}$,

(ii) ${ }^{p} j_{!}{ }^{p} j^{*}(\mathcal{T}) \subseteq \mathcal{T}$

(iii) ${ }^{p} j_{*}{ }^{p} j^{*}(\mathcal{F}) \subseteq \mathcal{F}$.

Proof. Item (1): We first check the orthogonality condition for $\left({ }^{p} i^{*}(\mathcal{T})\right.$, $\left.p_{i} !(\mathcal{F})\right)$. We have that

$$
\operatorname{Hom}_{\mathcal{A}_{\mathcal{D}}}\left({ }^{p} i^{*}(\mathcal{T}),{ }^{p} i^{!}(\mathcal{F})\right)=\operatorname{Hom}_{\mathcal{A}_{\mathcal{D}}}\left({ }^{p} i_{*}{ }^{p} i^{*}(\mathcal{T}),{ }^{p} i_{*}{ }^{p} i^{!}(\mathcal{F})\right)
$$

since ${ }^{p} i_{*}$ is fully faithful. Consider the exact sequence (6.2) with $A$ in $\mathcal{T}$ and apply to it the functor $\operatorname{Hom}_{\mathcal{A}_{\mathcal{D}}}(-, B)$ for some $B$ in $\mathcal{F}$. Since $\operatorname{Hom}_{\mathcal{A}_{\mathcal{D}}}(A, B)=0$, we conclude that $\operatorname{Hom}\left({ }^{p} i_{*}{ }^{p} i^{*}(A), B\right)=0$, that is, ${ }^{p} i_{*}{ }^{p} i^{*}(\mathcal{T}) \subseteq \mathcal{T}$. Similarly, we have ${ }^{p} i_{*}{ }^{p} i^{!}(\mathcal{F}) \subseteq \mathcal{F}$ and, thus, Hom $\mathcal{A}_{\mathcal{D}}\left({ }^{p} i_{*}{ }^{p} i^{*}(\mathcal{T})\right.$, $\left.p_{i_{*}} i^{!}(\mathcal{F})\right)=0$, as wanted.

Second, we produce a suitable short exact sequence for any object in $\mathcal{A}_{\mathcal{D}}$. By [5, Remarque 1.4.17.1], ${ }^{p} i_{*}\left(\mathcal{A}_{\mathcal{Y}}\right)$ is a Serre subcategory of $\mathcal{A}_{\mathcal{D}}$, that is, for any short exact sequence in $\mathcal{A}_{\mathcal{D}}$

$$
0 \rightarrow K \rightarrow M \rightarrow L \rightarrow 0
$$

$M$ lies in the ${ }^{p} i_{*}\left(\mathcal{A}_{\mathcal{Y}}\right)$ if and only if $K$ and $L$ lie there also. Let $M$ lie in $\mathcal{A}_{\mathcal{Y}}$, and consider a short exact sequence

$$
0 \rightarrow \widetilde{M_{1}} \rightarrow{ }^{p} i_{*}(M) \rightarrow \widetilde{M_{2}} \rightarrow 0
$$

in $\mathcal{A}_{\mathcal{D}}$ with $\widetilde{M}_{1} \in \mathcal{T}$ and $\widetilde{M}_{2} \in \mathcal{F}$ (which exists since $(\mathcal{T}, \mathcal{F}$ ) is a torsion pair in $\mathcal{D})$. Since ${ }^{p} i_{*}\left(\mathcal{A}_{\mathcal{Y}}\right)$ is a Serre subcategory of $\mathcal{A}_{\mathcal{D}}$, there exist $M_{1}$ and $M_{2}$ in $\mathcal{A} \mathcal{Y}$ such that $\widetilde{M}_{i}={ }^{p} i_{*}\left(M_{i}\right)$ for $i=1,2$. Now, ${ }^{p} i_{*}$ is an exact full embedding (see Remark 6.1) and, thus, we get a short exact sequence in $\mathcal{A}_{\mathcal{Y}}$

$$
0 \rightarrow M_{1} \rightarrow M \rightarrow M_{2} \rightarrow 0
$$


with $M_{1} \cong p i^{* p} i_{*}\left(M_{1}\right)={ }^{p} i^{*}\left(\widetilde{M}_{1}\right)$ lying in $i^{*}(\mathcal{T})$ and $M_{2} \cong p i^{! p} i_{*}\left(M_{2}\right)=$ $p_{i} !\left(\widetilde{M_{2}}\right)$ lying in $p^{!}(\mathcal{F})$.

Item (2): By Remark 6.1, the functor ${ }^{p} j^{*}: \mathcal{A}_{\mathcal{D}} \rightarrow \mathcal{A}_{\mathcal{X}}$ is exact. Hence, $\left({ }^{p} j^{*}(\mathcal{T}),{ }^{p} j^{*}(\mathcal{F})\right)$ is a torsion pair in $\mathcal{A}_{\mathcal{X}}$ if and only these classes are orthogonal, that is, $\operatorname{Hom}_{\mathcal{A}_{\mathcal{X}}}\left({ }^{p} j^{*}(\mathcal{T}),{ }^{p} j^{*}(\mathcal{F})\right)=0$. Using the adjunctions $\left({ }^{p} j_{!},{ }^{p} j^{*}\right)$ and $\left({ }^{p} j^{*},{ }^{p} j_{*}\right)$, this holds if and only if $\operatorname{Hom}_{\mathcal{A}_{\mathcal{D}}}\left({ }^{p} j^{p} j^{*}(\mathcal{T}), \mathcal{F}\right)=0$ (equivalent to (ii)) and if and only if $\operatorname{Hom}_{\mathcal{A}_{\mathcal{D}}}\left(\mathcal{T},{ }^{p} j_{*}{ }^{p} j^{*}(\mathcal{F})\right)=0$ (equivalent to (iii)).

When the conditions of item (2) of the lemma are fulfilled, we say that $(\mathcal{T}, \mathcal{F})$ is compatible with the recollement (6.1). We will see that this compatibility condition is precisely the requirement on a torsion pair so that the corresponding HRS-tilt of a glued t-structure is also obtained by gluing.

Proposition 6.3. A torsion pair $(\mathcal{T}, \mathcal{F})$ in $\mathcal{A}_{\mathcal{D}}$ is compatible with respect to (6.1) if and only if the corresponding HRS-tilt of $\left(\mathcal{D}^{\leq 0}, \mathcal{D}^{\geq 0}\right)$ is a $t$ structure glued with respect to the recollement (2.1).

Proof. Let $(\mathcal{T}, \mathcal{F})$ be a torsion pair in $\mathcal{A}_{\mathcal{D}}$, and let $\left(\widetilde{\mathcal{D}}^{\leq 0}, \widetilde{\mathcal{D}}^{\geq 0}\right)$ be the corresponding HRS-tilt, that is,

$$
\begin{aligned}
& \widetilde{\mathcal{D}}^{\leq 0}=\left\{X \in \mathcal{D} \mid H^{0}(X) \in \mathcal{T}, H^{i}(X)=0, \forall i>0\right\}, \\
& \widetilde{\mathcal{D}}^{\geq 0}=\left\{X \in \mathcal{D} \mid H^{-1}(X) \in \mathcal{F}, H^{i}(X)=0, \forall i<-1\right\},
\end{aligned}
$$

where $H^{i}, i \in \mathbb{Z}$, denote the cohomological functors of the t-structure $\left(\mathcal{D}^{\leq 0}, \mathcal{D}^{\geq 0}\right)$. By [5], the HRS-tilt is glued if and only if $j ! j^{*}\left(\widetilde{\mathcal{D}}^{\leq 0}\right) \subseteq \widetilde{\mathcal{D}}^{\leq 0}$. Denote by $\tau^{\leq-1}$ the truncation at -1 associated to $\left(\mathcal{D}^{\leq 0}, \mathcal{D}^{\geq 0}\right)$. For any $X \in \widetilde{\mathcal{D}}^{\leq 0}$, there is a triangle in $\mathcal{D}$ of the form

$$
\tau^{\leq-1} X \rightarrow X \rightarrow H^{0} X \rightarrow\left(\tau^{-1} X\right)[1]
$$

since, by definition, $\widetilde{\mathcal{D}} \leq 0 \subseteq \mathcal{D}^{\leq 0}$. Applying to it the functor $j ! j^{*}$, we get another triangle

$$
j ! j^{*}\left(\tau^{\leq-1} X\right) \rightarrow j ! j^{*}(X) \rightarrow j ! j^{*}\left(H^{0} X\right) \rightarrow j ! j^{*}\left(\tau^{\leq-1} X\right)[1]
$$

Since $\left(\mathcal{D}^{\leq 0}, \mathcal{D}^{\geq 0}\right)$ is glued, $j_{!} j^{*}\left(\mathcal{D}^{\leq-1}\right) \subseteq \mathcal{D}^{\leq-1} \subseteq \widetilde{\mathcal{D}} \leq 0$. Hence, $j_{!} j^{*}\left(\tau \tau^{\leq-1} X\right)$ lies in $\widetilde{\mathcal{D}} \leq 0$ and, thus, $j_{!} j^{*}(X)$ lies in $\widetilde{\mathcal{D}} \leq 0$ if and only if $j_{!} j^{*}\left(H^{0} X\right)$ lies in $\widetilde{\mathcal{D}} \leq 0$. Since $X$ is arbitrary, this means precisely that $\left(\widetilde{\mathcal{D}}^{\leq 0}, \widetilde{\mathcal{D}}^{\geq 0}\right)$ can be restricted if and only if the torsion pair $(\mathcal{T}, \mathcal{F})$ is compatible with (6.1). 
The question that naturally follows is whether, under the compatibility condition on the torsion pair in $\mathcal{D}$, the restrictions of the HRS-tilt of a glued t-structure in $\mathcal{D}$ are precisely the HRS-tilts of the restricted t-structures on $\mathcal{Y}$ and $\mathcal{X}$ with respect to the restricted torsion pairs.

TheOREM 6.4. Let $(\mathcal{T}, \mathcal{F})$ be a torsion pair in $\mathcal{A}_{\mathcal{D}}$, compatible with (6.1) and with restrictions $\left(\mathcal{T}_{\mathcal{Y}}, \mathcal{F}_{\mathcal{Y}}\right)$ and $\left(\mathcal{T}_{\mathcal{X}}, \mathcal{F}_{\mathcal{X}}\right)$ in $\mathcal{A}_{\mathcal{Y}}$ and $\mathcal{A}_{\mathcal{X}}$, respectively. Let $(\tilde{\mathcal{Y}} \leq 0, \tilde{\mathcal{Y}} \geq 0)$ and $\left(\tilde{\mathcal{X}} \leq 0, \widetilde{\mathcal{X}}^{\geq 0}\right)$ be the corresponding HRS-tilts of $(\mathcal{Y} \leq 0, \mathcal{Y} \geq 0)$ and $(\mathcal{X} \leq 0, \mathcal{X} \geq 0)$, respectively. Then the HRS-tilt $\left(\widetilde{\mathcal{D}}^{\leq 0}, \widetilde{\mathcal{D}}^{\geq 0}\right)$ is obtained by gluing $(\widetilde{\mathcal{X}} \leq 0, \tilde{\mathcal{X}} \geq 0)$ and $(\widetilde{\mathcal{Y}} \leq 0, \widetilde{\mathcal{Y}} \geq 0)$ with respect to the recollement (2.1).

Proof. Let $H_{\mathcal{D}}^{i}, H_{\mathcal{X}}^{i}$, and $H_{\mathcal{Y}}^{i}, i \in \mathbb{Z}$, denote the cohomological functors associated with $\left(\mathcal{D}^{\leq 0}, \mathcal{D}^{\geq 0}\right),\left(\mathcal{X}^{\leq 0}, \mathcal{X}^{\geq 0}\right)$, and $\left(\mathcal{Y}^{\leq 0}, \mathcal{Y}^{\geq 0}\right)$, respectively. We have to show that the following hold:

(i) $j^{*}(\widetilde{\mathcal{D}} \leq 0)=\widetilde{\mathcal{X}} \leq 0, j^{*}(\widetilde{\mathcal{D}} \geq 0)=\widetilde{\mathcal{X}} \geq 0$,

(ii) $i^{*}(\widetilde{\mathcal{D}} \leq 0)=\widetilde{\mathcal{Y}} \leq 0, i^{!}\left(\widetilde{\mathcal{D}}^{\geq 0}\right)=\widetilde{\mathcal{Y}} \geq 0$.

By definition, we have

$$
j^{*}\left(\widetilde{\mathcal{D}}^{\leq 0}\right)=\left\{j^{*} X \mid X \in \mathcal{D}, H_{\mathcal{D}}^{0}(X) \in \mathcal{T}, H_{\mathcal{D}}^{i}(X)=0, \forall i>0\right\},
$$

and, since $j^{*}$ is t-exact (see $\left.[5]\right), H_{\mathcal{X}}^{i}\left(j^{*} X\right)=j^{*}\left(H_{\mathcal{D}}^{i}(X)\right)$, thus showing that

$$
j^{*}\left(\widetilde{\mathcal{D}}^{\leq 0}\right) \subseteq\left\{j^{*} X \mid X \in \mathcal{D}, H_{\mathcal{X}}^{0}\left(j^{*} X\right) \in j^{*} \mathcal{T}, H_{\mathcal{X}}^{i}\left(j^{*} X\right)=0, \forall i>0\right\}=\tilde{\mathcal{X}}^{\leq 0} .
$$

Similarly, we get that

$$
j^{*}\left(\widetilde{\mathcal{D}}^{\geq 0}\right) \subseteq\left\{j^{*} X \mid X \in \mathcal{D}, H_{\mathcal{X}}^{-1}\left(j^{*} X\right) \in j^{*} \mathcal{T}, H_{\mathcal{X}}^{i}\left(j^{*} X\right)=0, \forall i<-1\right\}=\widetilde{\mathcal{X}}^{\geq 0} .
$$

Since $(\mathcal{T}, \mathcal{F})$ is compatible with the recollement, by Proposition 6.3, the HRS-tilt $\left(\widetilde{\mathcal{D}}^{\leq 0}, \widetilde{\mathcal{D}}^{\geq 0}\right)$ can be restricted and, thus, $\left(j^{*}\left(\widetilde{\mathcal{D}}^{\leq 0}\right), j^{*}\left(\widetilde{\mathcal{D}}^{\geq 0}\right)\right)$ is a t-structure in $\mathcal{X}$. Since $j^{*}(\widetilde{\mathcal{D}} \leq 0) \subseteq \tilde{\mathcal{X}} \leq 0$, we also have that $j^{*}\left(\widetilde{\mathcal{D}}^{\geq 0}\right) \supseteq \widetilde{\mathcal{X}}^{\geq 0}$ and, thus, $j^{*}\left(\widetilde{\mathcal{D}}^{\geq 0}\right)=\widetilde{\mathcal{X}}^{\geq 0}$. This proves (i).

To prove (ii), observe that $i^{*}$ is right t-exact (i.e., $i^{*}\left(\mathcal{D}^{\leq 0}\right) \subseteq \mathcal{Y}^{\leq 0}$ ) and that

$$
i^{*}\left(\widetilde{\mathcal{D}}^{\leq 0}\right)=\left\{i^{*} X \mid X \in \mathcal{D}, H_{\mathcal{D}}^{0}(X) \in \mathcal{T}, H_{\mathcal{D}}^{i}(X)=0, \forall i>0\right\}
$$

By [5, Proposition 1.3.17(ii)], for $X$ in $\widetilde{\mathcal{D}}^{\leq 0} \subseteq \mathcal{D}^{\leq 0}$, we have $H_{\mathcal{Y}}^{0}\left(i^{*} X\right)=$ ${ }^{p} i^{*} H_{\mathcal{D}}^{0}(X)$ which belongs to ${ }^{*} i^{*}(\mathcal{T})$. Since $i^{*}(\widetilde{\mathcal{D}} \leq 0) \subseteq i^{*}(\mathcal{D} \leq 0) \subseteq \mathcal{Y} \leq 0$, we get that $H_{\mathcal{Y}}^{i}\left(i^{*} \widetilde{\mathcal{D}} \leq 0\right)=0$, for all $i>0$, and thus

$$
i^{*}\left(\widetilde{\mathcal{D}}^{\leq 0}\right) \subseteq\left\{i^{*} X \mid X \in \mathcal{D}, H_{\mathcal{Y}}^{0}\left(i^{*} X\right) \in{ }^{p} i^{*} \mathcal{T}, H_{\mathcal{Y}}^{i}\left(i^{*} X\right)=0, \forall i>0\right\}=\widetilde{\mathcal{Y}} \leq 0
$$


Analogously, we may conclude that

$$
i^{*}\left(\widetilde{\mathcal{D}}^{\geq 0}\right) \subseteq\left\{i^{*} X \mid X \in \mathcal{D}, H_{\mathcal{Y}}^{-1}\left(i^{*} X\right) \in{ }^{p} i^{*} \mathcal{F}, H_{\mathcal{Y}}^{i}\left(i^{*} X\right)=0, \forall i<-1\right\}=\widetilde{\mathcal{Y}}^{\geq 0}
$$

The same argument as before shows that an equality holds, as wanted.

The previous result showed that an HRS-tilt with respect to a compatible torsion pair is glued. We may now ask whether HRS-tilts on the sides of the recollement (2.1) glue to an HRS-tilt in the middle. The following proposition answers this question positively.

Proposition 6.5. Let $\left(\mathcal{T}_{\mathcal{Y}}, \mathcal{F}_{\mathcal{Y}}\right)$ and $\left(\mathcal{T}_{\mathcal{X}}, \mathcal{F}_{\mathcal{X}}\right)$ be torsion pairs in $\mathcal{A}_{\mathcal{Y}}$ and $\mathcal{A}_{\mathcal{X}}$, respectively. Then the pair $(\mathcal{T}, \mathcal{F})$ defined by

$$
\begin{aligned}
& \mathcal{T}=\left\{A \in \mathcal{A}_{\mathcal{D}} \mid{ }^{p} i^{*} A \in \mathcal{T}_{\mathcal{Y}},{ }^{p} j^{*} A \in \mathcal{T}_{\mathcal{X}}\right\}, \\
& \mathcal{F}=\left\{A \in \mathcal{A}_{\mathcal{D}} \mid{ }^{p} i^{!} A \in \mathcal{F}_{\mathcal{Y}},{ }^{p} j^{*} A \in \mathcal{F}_{\mathcal{X}}\right\}
\end{aligned}
$$

is a torsion pair in $\mathcal{A}_{\mathcal{D}}$, compatible with (6.1). Moreover, its restrictions are given by $\left(\mathcal{T}_{\mathcal{X}}, \mathcal{F}_{\mathcal{X}}\right)$ and $\left(\mathcal{T}_{\mathcal{Y}}, \mathcal{F}_{\mathcal{Y}}\right)$, and the HRS-tilt of $\left(\mathcal{D}^{\leq 0}, \mathcal{D}^{\geq 0}\right)$ with respect to $(\mathcal{T}, \mathcal{F})$ is the gluing of the HRS-tilts of $\left(\mathcal{Y}^{\leq 0}, \mathcal{Y} \geq 0\right)$ and $(\mathcal{X} \leq 0, \mathcal{X} \geq 0)$ with respect to $\left(\mathcal{T}_{\mathcal{Y}}, \mathcal{F}_{\mathcal{Y}}\right)$ and $\left(\mathcal{T}_{\mathcal{X}}, \mathcal{F}_{\mathcal{X}}\right)$, respectively.

Proof. We will use Remark 2.9 to show that $(\mathcal{T}, \mathcal{F})$ is a torsion pair, that is, we will show that the pair $\left(\mathcal{D}_{(\mathcal{T}, \mathcal{F})}^{\leq 0}, \mathcal{D}_{(\mathcal{T}, \mathcal{F})}^{\geq 0}\right)$ formally defined as the HRS-tilt of $\left(\mathcal{D}^{\leq 0}, \mathcal{D}^{\geq 0}\right)$ with respect to $(\mathcal{T}, \mathcal{F})$ is a t-structure (satisfying $\left.\mathcal{D}^{\leq-1} \subseteq \mathcal{D}_{(\mathcal{T}, \mathcal{F})}^{\leq 0} \subseteq \mathcal{D}^{\leq 0}\right)$. Denote by $\left(\widetilde{\mathcal{Y}}^{\leq 0}, \tilde{\mathcal{Y}}^{\geq 0}\right)$ and $\left(\widetilde{\mathcal{X}}^{\leq 0}, \widetilde{\mathcal{X}}^{\geq 0}\right)$ the HRStilts of $(\mathcal{Y} \leq 0, \mathcal{Y} \geq 0)$ and $(\mathcal{X} \leq 0, \mathcal{X} \geq 0)$ at $\left(\mathcal{T}_{\mathcal{Y}}, \mathcal{F}_{\mathcal{Y}}\right)$ and $\left(\mathcal{T}_{\mathcal{X}}, \mathcal{F}_{\mathcal{X}}\right)$, respectively, and let $\left(\widetilde{\mathcal{D}}^{\leq 0}, \widetilde{\mathcal{D}}^{\geq 0}\right)$ be the gluing of $\left(\widetilde{\mathcal{Y}}^{\leq 0}, \widetilde{\mathcal{Y}}^{\geq 0}\right)$ and $\left(\widetilde{\mathcal{X}} \leq 0, \widetilde{\mathcal{X}}^{\geq 0}\right)$ with respect to the fixed recollement, that is,

$$
\begin{aligned}
& \widetilde{\mathcal{D}}^{\leq 0}=\left\{X \in \mathcal{D} \mid i^{*}(X) \in \widetilde{\mathcal{Y}}^{\leq 0}, j^{*}(X) \in \widetilde{\mathcal{X}}^{\leq 0}\right\}, \\
& \widetilde{\mathcal{D}}^{\geq 0}=\left\{X \in \mathcal{D} \mid i^{!}(X) \in \widetilde{\mathcal{Y}}^{\geq 0}, j^{*}(X) \in \widetilde{\mathcal{X}}^{\geq 0}\right\} .
\end{aligned}
$$

We will show that $\left(\widetilde{\mathcal{D}}^{\leq 0}, \widetilde{\mathcal{D}}^{\geq 0}\right)=\left(\mathcal{D}_{(\mathcal{T}, \mathcal{F})}^{\leq 0}, \mathcal{D}_{(\mathcal{T}, \mathcal{F})}^{\geq 0}\right)$. As usual, $H_{\mathcal{D}}^{i}, H_{\mathcal{X}}^{i}$, and $H_{\mathcal{Y}}^{i}, i \in \mathbb{Z}$, denote the cohomological functors associated with $\left(\mathcal{D}^{\leq 0}, \mathcal{D}^{\geq 0}\right)$, $\left(\mathcal{X}^{\leq 0}, \mathcal{X}^{\geq 0}\right)$, and $\left(\mathcal{Y}^{\leq 0}, \mathcal{Y}^{\geq 0}\right)$, respectively.

First we show that $\mathcal{D}_{(\mathcal{T}, \mathcal{F})}^{\leq 0}$ is a subcategory of $\widetilde{\mathcal{D}}^{\leq 0}$. Take $X$ in $\mathcal{D}_{(\mathcal{T}, \mathcal{F})}^{\leq 0}$ $\left(\subseteq \mathcal{D}^{\leq 0}\right)$. Since $i^{*}$ is right t-exact, $i^{*}(X)$ lies in $\mathcal{Y} \leq 0$, that is, $H_{\mathcal{Y}}^{i}\left(i^{*} X\right)=$ 0 , for all $i>0$. By [5, Proposition 1.3.17(ii)], $H_{\mathcal{Y}}^{0}\left(i^{*} X\right)={ }^{p} i^{*} H_{\mathcal{D}}^{0}(X)$ lies 
in $p^{*}(\mathcal{T}) \subseteq \mathcal{T} \mathcal{Y}$, showing that $i^{*}(X) \in \widetilde{\mathcal{Y}} \leq 0$. Since $j^{*}$ is t-exact, we have $H_{\mathcal{X}}^{i}\left(j^{*} X\right)=j^{*} H_{\mathcal{D}}^{i}(X)=0$, for all $i>0$, and $H_{\mathcal{X}}^{0}\left(j^{*} X\right)=j^{*} H_{\mathcal{D}}^{0}(X)$ lies in $j^{*}(\mathcal{T}) \subseteq \mathcal{T}_{\mathcal{X}}$, that is, $j^{*} X$ lies in $\widetilde{\mathcal{X}} \leq 0$ and, hence, $X$ lies in $\widetilde{\mathcal{D}} \leq 0$.

Now we prove that $\widetilde{\mathcal{D}}^{\leq 0}$ is a subcategory of $\mathcal{D}_{(\mathcal{T}, \mathcal{F})}^{\leq 0}$. Take $X$ in $\widetilde{\mathcal{D}}^{\leq 0}$. By definition of HRS-tilt, we have $\tilde{\mathcal{Y}} \leq 0 \subset \mathcal{Y} \leq 0$ and $\widetilde{\mathcal{X}} \leq 0 \subseteq \mathcal{X} \leq 0$. This implies that $\widetilde{\mathcal{D}}^{\leq 0} \subseteq \mathcal{D}^{\leq 0}$ and, hence, $H_{\mathcal{D}}^{i}(X)=0$, for all $i>0$. By [5, Proposition 1.3.17(ii)], we have ${ }^{p} i^{*} H_{\mathcal{D}}^{0}(X)=H_{\mathcal{Y}}^{0}\left(i^{*} X\right)$ which belongs to $\mathcal{T}_{\mathcal{Y}}$. By the t-exactness of $j^{*}$, we have ${ }^{p} j^{*} H_{\mathcal{D}}^{0}(X)=H_{\mathcal{X}}^{0}\left(j^{*} X\right)$ which, thus, belongs to $\mathcal{T}_{\mathcal{X}}$. This means that, by definition of $\mathcal{T}, H_{\mathcal{D}}^{0}(X)$ belongs to $\mathcal{T}$. Thus $X$ belongs to $\widetilde{\mathcal{D}}^{\leq 0}$ and $\widetilde{\mathcal{D}} \leq 0=\mathcal{D}_{(\mathcal{T}, \mathcal{F})}^{\leq 0}$, as wanted.

It remains to show that $(\mathcal{T}, \mathcal{F})$ is compatible and that the restrictions are as expected. By Lemma 6.2, we need to show that ${ }^{p} j_{!}{ }^{p} j^{*} \mathcal{T} \subseteq \mathcal{T}$. This is true since, for any $A$ in $\mathcal{T},{ }^{p} i^{* p}{ }_{j !}{ }^{p} j^{*} A=0$ and ${ }^{p} j^{* p} j_{!}^{p} j^{*} A={ }^{p} j^{*} A \subseteq \mathcal{T}_{\mathcal{X}}$. It follows that $\left({ }^{p} i^{*}(\mathcal{T}),{ }^{p} i^{!}(\mathcal{F})\right)$ and $\left({ }^{p} j^{*}(\mathcal{T}),{ }^{p} j^{*}(\mathcal{F})\right)$ are torsion pairs in $\mathcal{A}_{\mathcal{Y}}$ and $\mathcal{A}_{\mathcal{X}}$, respectively. On the other hand, by definition of $(\mathcal{T}, \mathcal{F})$, we have

$$
\begin{aligned}
{ }^{p} i^{*}(\mathcal{T}) \subseteq \mathcal{T}_{\mathcal{Y}}, & p i^{!}(\mathcal{F}) \subseteq \mathcal{F}_{\mathcal{Y}}, \\
{ }^{p} j^{*}(\mathcal{T}) \subseteq \mathcal{T}_{\mathcal{X}}, & { }^{p} j^{*}(\mathcal{F}) \subseteq \mathcal{F}_{\mathcal{X}},
\end{aligned}
$$

and, thus, the inclusions are actually equalities, as desired.

We illustrate Proposition 6.5 with a simple example.

EXAMPLE 6.6. We adopt the notation in Example 5.3 and consider the standard recollement there. Let $\left(\mathcal{D}^{\leq 0}, \mathcal{D}^{\geq 0}\right)$ be the standard t-structure on $\mathcal{D}^{b}(R)$, which is depicted in the Auslander-Reiten quiver of $\mathcal{D}^{b}(R)$ as

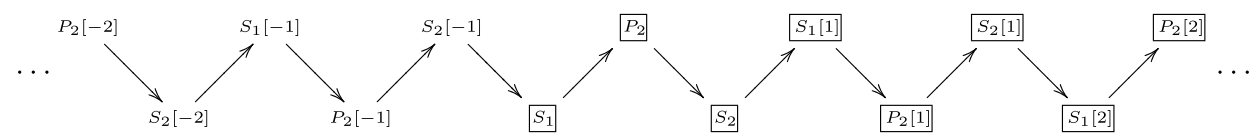

where the objects in the boxes belong to the aisle $\mathcal{D} \leq 0$. This t-structure restricts to standard t-structures $(\mathcal{X} \leq 0, \mathcal{X} \geq 0)$ and $(\mathcal{Y} \leq 0, \mathcal{Y} \geq 0)$ on $\mathcal{D}^{b}(e R e)$ and $\mathcal{D}^{b}(R / R e R)$, respectively. Recall that both $e R e$ and $R / R e R$ are isomorphic to $\mathbb{K}$. Consequently, all bounded t-structures on $\mathcal{D}^{b}(e R e)$ and $\mathcal{D}^{b}(R / R e R)$ are shifts of the standard ones, and all mutation torsion pairs of $\bmod -e R e$ and $\bmod -R / R e R$ are trivial torsion pairs.

The HRS-tilt of $(\mathcal{X} \leq 0, \mathcal{X} \geq 0)$ with respect to the torsion pair $(0, \bmod -e R e)$ is $(\mathcal{X} \leq 0, \mathcal{X} \geq 0)$, and the HRS-tilt of $\left(\mathcal{Y}^{\leq 0}, \mathcal{Y}^{\geq 0}\right)$ with respect to the torsion 
pair $(\bmod -R / \operatorname{Re} R, 0)$ is $\left(\mathcal{Y} \leq 0[1], \mathcal{Y}^{\geq 0}[1]\right)$. These two new t-structures are glued, via the standard recollement, to a t-structure $\left(\mathcal{D}^{\prime \leq 0}, \mathcal{D}^{\prime \geq 0}\right)$ on $\mathcal{D}^{b}(R)$, which is depicted as

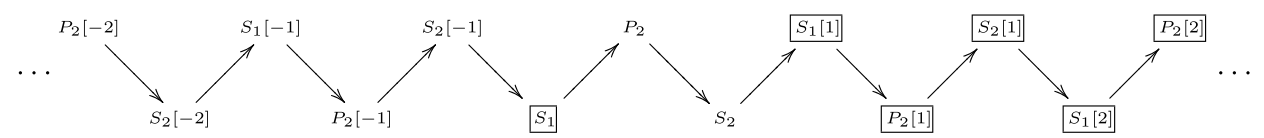

where the objects in the boxes belong to the aisle $\mathcal{D}^{\prime \leq 0}$. The heart of $\left(\mathcal{D}^{\prime \leq 0}, \mathcal{D}^{\prime \geq 0}\right)$ is $\operatorname{add}\left(S_{1} \oplus S_{2}[1]\right)$. It is easy to check that $\left(\mathcal{D}^{\prime \leq 0}, \mathcal{D}^{\prime \geq 0}\right)$ is the HRS-tilt of $\left(\mathcal{D}^{\leq 0}, \mathcal{D}^{\geq 0}\right)$ at the torsion pair $\left(\operatorname{add}\left(S_{1}\right)\right.$, add $\left.\left(S_{2}\right)\right)$, which is glued from the torsion pairs $(0, \bmod -e R e)$ and $(\bmod -R / R e R, 0)$.

By Section 2.4, we know that irreducible silting mutations correspond to HRS-tilts (see Theorem 2.11) with respect to mutation torsion pairs in the heart (i.e., torsion pairs in which either the torsion class or the torsion-free class are given by the additive closure of a simple object). We analyze when such torsion pairs are compatible with (6.1).

Remark 6.7. Recall that there is a functor $j_{!_{*}}: \mathcal{A}_{\mathcal{X}} \rightarrow \mathcal{A}_{\mathcal{D}}$, called the intermediate image functor, such that every simple object in $\mathcal{A}_{\mathcal{D}}$ is either of the form $i_{*} S$ for some simple object in $\mathcal{A}_{\mathcal{Y}}$ or of the form $j_{!_{*}} S$ for some simple object in $\mathcal{A}_{\mathcal{X}}$. (For details, check [5, Section 1.4].)

Proposition 6.8. Suppose that $\mathcal{A}_{\mathcal{D}}$ is a length category. Let $(\mathcal{T}, \mathcal{F})$ be a mutation torsion pair in $\mathcal{A}_{\mathcal{D}}$. Then $(\mathcal{T}, \mathcal{F})$ is compatible with the recollement (6.1) if and only if one of the following holds:

(1) $\mathcal{T}=\operatorname{add}\left(i_{*} S_{Y}\right)$ for some simple object $S_{Y}$ in $\mathcal{A}_{\mathcal{y}}$,

(2) $\mathcal{F}=\operatorname{add}\left(i_{*} S_{Y}\right)$ for some simple object $S_{Y}$ in $\mathcal{A}_{\mathcal{Y}}$,

(3) there is a simple object $S_{X}$ in $\mathcal{A}_{\mathcal{X}}$ such that ${ }^{p}{ }_{j_{1}} S_{X}$ is simple and $\mathcal{T}=$ $\operatorname{add}\left({ }^{p} j_{!} S_{X}\right)$,

(4) there is a simple object $S_{X}$ in $\mathcal{A}_{\mathcal{X}}$ such that ${ }^{p} j_{*} S_{X}$ is simple and $\mathcal{F}=$ $\operatorname{add}\left({ }^{p} j_{*} S_{X}\right)$.

Moreover, in each case, the restrictions are a trivial torsion pair on one side and a mutation torsion pair on the other.

Proof. This can be shown using Lemma 6.2 on a case-by-case analysis of the possible mutation torsion pairs in $\mathcal{A}_{\mathcal{D}}$. Using Remark 6.7 we get the following four cases, where $S_{Y}$ is a simple object in $\mathcal{A}_{\mathcal{Y}}$ and $S_{X}$ is a simple object in $\mathcal{A}_{\mathcal{X}}$. 
(1) Suppose that $\mathcal{T}=\operatorname{add}\left(i_{*} S_{Y}\right)$. Since $j^{*} i_{*}=0$, we have that ${ }^{p} j_{!} j^{*} \mathcal{T}=0$ and, thus, the torsion pair $(\mathcal{T}, \mathcal{F})$ is compatible with the recollement. The restriction to $\mathcal{X}$ is $(0, \mathcal{X})$ and the restriction to $\mathcal{Y}$ is the mutation torsion pair with torsion class $\mathcal{T}_{\mathcal{Y}}=\operatorname{add}\left(S_{Y}\right)$.

(2) Suppose that $\mathcal{F}=\operatorname{add}\left(i_{*} S_{Y}\right)$. Since $j^{*} i_{*}=0$, we have that ${ }^{p} j_{*} j^{*} \mathcal{T}=0$ and, thus, the torsion pair $(\mathcal{T}, \mathcal{F})$ is compatible with the recollement. The restriction to $\mathcal{X}$ is $(\mathcal{X}, 0)$ and the restriction to $\mathcal{Y}$ is the mutation torsion pair with torsion-free class $\mathcal{F}_{\mathcal{Y}}=\operatorname{add}\left(S_{Y}\right)$.

(3) Suppose that $\mathcal{T}=\operatorname{add}\left(j_{! *} S_{X}\right)$. By Lemma 6.2, the torsion pair $(\mathcal{T}, \mathcal{F})$ is compatible with the recollement if and only if ${ }^{p} j_{!} j^{*}\left(j_{! *} S_{X}\right)$ lies in $\operatorname{add}\left(j_{! *} S_{X}\right)$. Since $j^{*} j_{\text {!* }}$ is naturally equivalent to the identity functor, this amounts to ${ }^{p} j_{!} S_{X}$ lying in $\operatorname{add}\left(j_{\text {!* }} S_{X}\right)$, which is equivalent to ${ }^{p} j_{!} S_{X} \cong j_{\text {!* }} S_{X}$ because $j^{* p} j_{\text {! }}$ is also naturally equivalent to the identity functor. The last condition holds if and only if ${ }^{p} j_{!} S_{X}$ is simple. This follows from the fact that there is always a natural epimorphism ${ }^{p} j_{!} S_{X} \rightarrow$ $j_{!_{*}} S_{X}$. The restriction to $\mathcal{Y}$ is the pair $(0, \mathcal{Y})$ and the restriction to $\mathcal{X}$ is the mutation torsion pair given by the torsion class $\mathcal{T}_{\mathcal{X}}=\operatorname{add}\left(S_{X}\right)$.

(4) Suppose that $\mathcal{F}=\operatorname{add}\left(j_{!_{*}} S_{X}\right)$. By Lemma 6.2 , the torsion pair $(\mathcal{T}, \mathcal{F})$ is compatible with the recollement if and only if ${ }^{p} j_{*} j^{*}\left(j_{!_{*}} S_{X}\right)$ lies in $\operatorname{add}\left(j_{! *} S_{X}\right)$. Since $j^{*} j_{! *}$ is naturally equivalent to the identity functor, this amounts to ${ }^{p} j_{*} S_{X}$ lying in $\operatorname{add}\left(j_{!_{*}} S_{X}\right)$, which is equivalent to ${ }^{p} j_{*} S_{X} \cong j_{!_{*}} S_{X}$ because $j^{* p} j_{*}$ is also naturally equivalent to the identity functor. The last condition holds if and only if ${ }^{p} j_{*} S_{X}$ is simple. This follows from the fact that there is always a natural monomorphism $j_{!_{*}} S_{X} \rightarrow{ }^{p} j_{*} S_{X}$. The restriction to $\mathcal{Y}$ is the pair $(\mathcal{Y}, 0)$ and the restriction to $\mathcal{X}$ is the mutation torsion pair given by the torsion-free $\operatorname{class} \mathcal{F}_{\mathcal{X}}=\operatorname{add}\left(S_{X}\right)$.

The proof is complete.

Suppose now that $\mathcal{D}$ has a Serre functor. We finish this section with an observation on the compatibility of irreducible silting mutation with gluing (via the recollement $\mathcal{R}_{U}$, since our focus is on t-structures rather than co-tstructures). Note that the items (3) and (4) of Proposition 6.8 rarely occur, because, in general, neither ${ }^{p} j_{*} S_{X}$ nor ${ }^{p} j_{!} S_{Y}$ are simple in $\mathcal{A}_{\mathcal{D}}$. Translating items (1) and (2) of Proposition 6.8 in terms of irreducible silting mutation, we get the following corollary, which also appears in [1, Lemma 2.40].

Corollary 6.9 ([1, Lemma 2.40]). Let $Y$ and $X$ be the silting objects associated to the fixed bounded $t$-structures in $\mathcal{Y}$ and $\mathcal{X}$, respectively. Suppose 
that $Y^{\prime}$ is an indecomposable direct summand of $Y$. Let $Z$ be the glued silting object with respect to the recollement $\mathcal{R}_{U}$ (i.e., compatible with the gluing of $t$-structures via $\mathcal{R}$ ) of $Y$ and $X$. Then there is an indecomposable direct summand $Z^{\prime}$ of $K_{Y}$ such that the glued silting (with respect to $\mathcal{R}_{U}$ ) of $\mu_{Y^{\prime}}^{+} Y$ (resp., $\mu_{Y^{\prime}}^{-} Y$ ) and $X$ is precisely $\mu_{Z^{\prime}}^{+} Z$ (resp., $\mu_{Z^{\prime}}^{-} Z$ ).

Acknowledgments. The authors would like to thank David Pauksztello and Lutz Hille for helpful remarks and for a discussion which led to Example 4.4. Vitória's work was supported by DFG-Schwerpunktsprogramm Darstellungstheorie 1388 grant KO1281/7-1. Yang's work was supported by DFG-Schwerpunktsprogramm Darstellungstheorie 1388 grant KO1281/9-1.

\section{REFERENCES}

[1] T. Aihara and O. Iyama, Silting mutation in triangulated categories, J. Lond. Math. Soc. (2) 85 (2012), 633-668. MR 2927802. DOI 10.1112/jlms/jdr055.

[2] L. Angeleri Hügel, S. Koenig, and Q. Liu, Recollements and tilting objects, J. Pure Appl. Algebra 215 (2011), 420-438. MR 2738361. DOI 10.1016/j.jpaa.2010.04.027.

[3] - Jordan-Hölder theorems for derived module categories of piecewise hereditary algebras, J. Algebra 352 (2012), 361-381. MR 2862193. DOI 10.1016/j.jalgebra.2011.09.041.

[4] - On the uniqueness of stratifications of derived module categories, J. Algebra 359 (2012), 120-137. MR 2914629. DOI 10.1016/j.jalgebra.2012.02.022.

[5] A. A. Beilinson, J. Bernstein, and P. Deligne, "Faisceaux pervers" in Analysis and Topology on Singular Spaces, I (Luminy, 1981), Astérisque 100, Soc. Math. France, Paris, 1982, 5-171. MR 0751966.

[6] A. Beligiannis and I. Reiten, Homological and Homotopical Aspects of Torsion Theories, Mem. Amer. Math. Soc. 188, 2007. MR 2327478. DOI 10.1090/memo/0883.

[7] A. I. Bondal and M. M. Kapranov, Representable functors, Serre functors, and reconstructions (in Russian), Izv. Akad. Nauk SSSR Ser. Mat. 53, no. 6 (1989), 1183-1205, 1337; English translation in Math. USSR-Izv. 35, no. 3 (1990), 519-541. MR 1039961.

[8] M. V. Bondarko, Weight structures vs. t-structures; weight filtrations, spectral sequences, and complexes (for motives and in general), J. K-Theory 6 (2010), 387504. MR 2746283. DOI 10.1017/is010012005jkt083.

[9] T. Bridgeland, t-structures on some local Calabi-Yau varieties, J. Algebra 289 (2005), 453-483. MR 2142382. DOI 10.1016/j.jalgebra.2005.03.016.

[10] A. B. Buan, I. Reiten, and H. Thomas, Three kinds of mutation, J. Algebra 339 (2011), 97-113. MR 2811314. DOI 10.1016/j.jalgebra.2011.04.030.

[11] E. Cline, B. Parshall, and L. L. Scott, Algebraic stratification in representation categories, J. Algebra 117 (1988), 504-521. MR 0957457. DOI 10.1016/0021-8693(88)90123-8.

[12] - Stratifying Endomorphism Algebras, Mem. Amer. Math. Soc. 124 (1996), no. 591. MR 1350891. DOI 10.1090/memo/0591.

[13] V. Franjou and T. Pirashvili, Comparison of abelian categories recollements, Doc. Math. 9 (2004), 41-56. MR 2054979. 
[14] D. Happel, I. Reiten, and S. O. Smalø, Tilting in Abelian Categories and Quasitilted Algebras, Mem. Amer. Math. Soc. 120 (1996), no. 575. MR 1327209. DOI $10.1090 / \mathrm{memo} / 0575$.

[15] P. Jørgensen, Reflecting recollements, Osaka J. Math. 47 (2010), 209-213. MR 2666132.

[16] B. Keller and P. Nicolás, Cluster hearts and cluster tilting objects, in preparation.

[17] B. Keller and D. Vossieck, "Aisles in derived categories" in Deuxième Contact FrancoBelge en Algèbre (Faulx-les-Tombes, 1987), Bull. Soc. Math. Belg. Sér. A 40 (1988), 239-253. MR 0976638.

[18] S. Koenig and D. Yang, Silting objects, simple-minded collections, t-structures and co-t-structures for finite-dimensional algebras, Doc. Math. 19 (2014), 403-438. MR 3178243.

[19] S. Ladkani, Derived equivalences of triangular matrix rings arising from extensions of tilting modules, Algebr. Represent. Theory 14 (2011), 57-74. MR 2763291. DOI 10.1007/s10468-009-9175-0.

[20] Q. Liu and J. Vitória, t-structures via recollements for piecewise hereditary algebras, J. Pure Appl. Algebra 216 (2012), 837-849. MR 2864858. DOI 10.1016/j.jpaa.2011.08.010.

[21] O. Mendoza Hernández, E. C. Sáenz Valadez, V. Santiago Vargas, and M. J. Souto Salorio, Auslander-Buchweitz context and co-t-structures, Appl. Categ. Structures 21 (2013), 417-440. MR 3097052. DOI 10.1007/s10485-011-9271-2.

[22] D. Pauksztello, Compact corigid objects in triangulated categories and co-t-structures, Cent. Eur. J. Math. 6 (2008), 25-42. MR 2379950. DOI 10.2478/s11533-008-0003-2.

[23] I. Reiten and M. Van den Bergh, Noetherian hereditary abelian categories satisfying Serre duality, J. Amer. Math. Soc. 15 (2002), 295-366. MR 1887637. DOI 10.1090/S0894-0347-02-00387-9.

[24] J. Rickard, Morita theory for derived categories, J. Lond. Math. Soc. (2) 39 (1989), 436-456. MR 1002456. DOI 10.1112/jlms/s2-39.3.436.

[25] J. Rickard and A. Schofield, Cocovers and tilting modules, Math. Proc. Cambridge Philos. Soc. 106 (1989), 1-5. MR 0994075. DOI 10.1017/S0305004100067931.

[26] M. J. Souto Salorio and S. Trepode, T-structures on the bounded derived category of the Kronecker algebra, Appl. Categ. Structures 20 (2012), 513-529. MR 2957314. DOI 10.1007/s10485-011-9248-1.

[27] L. Alonso Tarrío, A. Jeremías López, and M. J. Souto Salorio, Construction of $t$ structures and equivalences of derived categories, Trans. Amer. Math. Soc. 355, no. 6 (2003), 2523-2543. MR 1974001. DOI 10.1090/S0002-9947-03-03261-6.

[28] Z. Wang, Nondegeneration and boundedness of t-structure induced by recollement, Xiamen Daxue Xuebao Ziran Kexue Ban 45 (2006), 10-13. MR 2193734.

[29] J. Woolf, Stability conditions, torsion theories and tilting, J. Lond. Math. Soc. (2) 82 (2010), 663-682. MR 2739061. DOI 10.1112/jlms/jdq035. 
Qunhua Liu

Institute of Mathematics

School of Mathematical Sciences

Nanjing Normal University

Nanjing 210023

People's Republic of China

05402@njnu.edu.cn

Jorge Vitória

Department of Computer Science-Sector of Mathematics University of Verona

I-37134 Verona

Italy

jorge.vitoria@univr.it

Dong Yang

Department of Mathematics

Nanjing University

Nanjing 210093

People's Republic of China

dongyang2002@gmail. com 